\title{
The algebraic independence of Weierstrass functions and some related numbers
}

\section{by}

W. Datik BroWnaWeILT(University Park, Penn.) and K. K. KUBota (Lexington, Ky.)

0. Introduction. This article develops and applies general criteria for the algebraic independence over $C(z)$ of functions of the form $\wp(\alpha z)$, $\zeta(\alpha z)$, and $\exp (\beta z)$. Such questions are interesting as function analogues of number theoretic transcendence questions. But they also lead to transcendence results for numbers.

The paper is divided into three main parts. The sections are somewhat independent, using only the statements but never the techniques of previous sections. The reader who is so inclined is invited to read only the sections interesting to him.

Section I. This first part generalizes some differential algebraic techniques of $J$. Ax ([1], [2]). He developed them to answer the power series analogue of a conjecture of $S$. Schanuel for the exponential function. We extend $A x$ 's theorem to include also the differential algebraic analogues of Abelian integrals of the first and second kinds. The proof of the central result follows the general outline of Ax's original proof, although of course some new elements are introduced. As an example of the results to be found at the end of this section, we note the fol lowing result:

For $i=1, \ldots, n$, let $\wp_{i}(z)$ be as Weierstrass elliptic function and $y_{i}$ be a power series without constant term. If $y_{1}, \ldots, y_{n}$ are C-linearly independent, then

$$
\text { tr. deg.c } C\left(y_{1}, \ldots, y_{n}, \wp_{1}\left(y_{1}\right), \ldots, \wp_{n}\left(y_{n}\right)\right) \geqslant n+1 .
$$

Section II. If for example the $\wp_{i}(z)$ have no complex multiplications, i.e. a ratio $\omega_{52} / \omega_{i 1}$ of fundamental periods is not imaginary quadratic, for $i=1, \ldots, n$, then the hypotheses in the preceding result that the $y_{i}$ be $\boldsymbol{C}$-linearly independent can be weakened to linear independence over the rational numbers $\boldsymbol{Q}$. In order to do this and to obtain the cor- 
responding result when complex multiplication is allowed, techniques from algebraic groups and Lie groups are introduced in this section. The main result is to be found in part $\mathrm{D}$. There seems to be some overlap of this section with reeent work of Ax [3], from which the idea of using the Lie correspondence for algebraic groups was taken.

Section III. We develop necessary and sufficient conditions for the hypotheses of Waldschmidt's general axiomatization ([21], $\S 5$ ) of transcendence proofs involving functions of the form $\wp(\alpha z), \zeta(\alpha z)$, and $\exp (\beta z)$. Several new transcendence results are deduced. Perhaps the most striking new results are those showing that of three numbers related to a Weierstrass elliptic function with complex multiplication, at least two are algebraically independent over $\boldsymbol{Q}$.

Let $\wp(z)$ be a Weierstrass elliptic funetion having its period ratio $=\tau=\omega_{2} / \omega_{1}$ imaginary quadratic and having its invariants $g_{2}, g_{3}$ algebraic. Assume that the complex number a is a cubic irrational. Then at least one of $\wp\left(\alpha \omega_{1}\right), \wp\left(\alpha^{2} \omega_{1}\right)$ is transcendental over $\boldsymbol{Q}\left(\omega_{1}\right)$.

This seems to be the first result published asserting the algebraic independence of two or more numbers connected with an elliptic function.

Appendix A shows that when the functions considered are algebraically dependent over $C$, then they are actually dependent over the "smallest" fields possible. In Appendix B, we show that when $g_{2}$ and $g_{3}$ are algebraic and $\wp(z)$ has complex multiplications, then $\omega_{1}, \eta_{1}$, and $\eta_{2}$ are linearly dependent over the algebraic numbers.

This work was begun at the NSF 1973 Summer Number Theory Institute at the University of Michigan. It is with pleasure that both authors thank the National Science Foundation for support that summer and thank D.J. Lewis, together with his colleagues and the staff at the University of Michigan, for making our stay so enjoyable.

The referee has called to our attention that the results of Appendices $A$ and $B$ can be derived with less calculation by the type of argument given by D.W. Masser in Elliptic Functions and Transcendence, Springer Lecture Notes Nr. 437, 1975, pp. 36, 37, 66 .

\section{DIFFERENTIAL ALGEBRA}

A. Derivations and differentials. From now on, all rings (and fields) are assumed to be integral domains with characteristic zero quotient fields, and all modules are assumed to be unitary.

1. Let us recall some standard facts $([15], \S 26, \S 27)$. If $B$ is an $A$-algebra and $M$ is a $B$-module, then an $A$-derivation of $B$ with values in $M$ is defined to be an $A$-linear map $D: B \rightarrow M$ such that for every pair $b_{1}, b_{2} \in B$, one has

$$
D\left(b_{1} b_{2}\right)=b_{2} D b_{1}+b_{1} D b_{2} \text {. }
$$

The $A$-derivations of $B$ with values in $M$ form a $B$-module which is denoted $\operatorname{Der}_{A}(B, M)$. The module of $A$-differentials of $B$ is a $B$-module $\Omega_{B / A}$ together with a derivation $d_{B \mid A}: B \rightarrow \Omega_{B \mid A}$ such that for any $A$-derivation $D: B \rightarrow M$, there is a unique $B$-linear map $D^{*}: \Omega_{B / A} \rightarrow M$ which makes the following triangle commute:

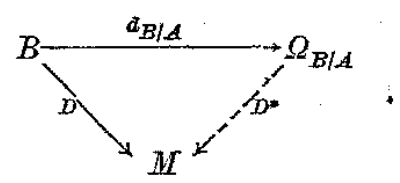

Thus the map $D \mapsto D^{*}$ is a $B$-isomorphism of $\operatorname{Der}_{\perp}(B, M)$ onto the "dual" Hom $_{B}\left(\Omega_{B / A}, M\right)$ of $\Omega_{B / A}$. Note that $D^{*}$ is just the map which replaces " $d$ " with " $D$ ", i.e.

$$
D^{*}\left(f d_{B / 4} z\right)=f D z \text {. }
$$

When the subscript " $B / A$ " is not needed for clarity, we shall usually write simply $d$ instead of $d_{B / A}$.

If $B$ and $A$ are fields, then tr.deg. ${ }_{A} B=\operatorname{dim}_{B} \Omega_{B / A}$ and in fact if $\left\{\alpha_{i}\right\}$ is a transcendence base of $B / A$, then $\left\{d \alpha_{i}\right\}$ is a $B$-basis of $\Omega_{B / A}$. In particular, if $k \subseteq K \subseteq L$ are fields, then one has a canonical injection $\Omega_{K / k} \otimes_{K} L \rightarrow Q_{L / K}$. Also, if $K$ and $k^{\prime}$ are fields which are algebraically free over $k$, then the canonical map $\Omega_{K / k} \otimes_{K} K k^{\prime} \rightarrow \Omega_{K k^{\prime} / k^{\prime}}$ is an isomorphism.

If $S$ is a multiplicative subset of the ring $B$ and $B_{S}$ is the localization of $B$ at $S$, then the map

$$
\Omega_{B / A} \otimes_{B} B_{S} \rightarrow Q_{B_{S} / A}
$$

is an isomorphism. Although in general, $\Omega_{B / A}$ may have some torsion, this does not oceur in case $B$ is a valuation ring over a field. More precisely:

LEMMA 1. If $B$ is a valuation ring containing a field $k$ and if $K$ is the quotient field of $B$, then the natural map

is injective.

$$
\Omega_{B / k} \rightarrow \Omega_{B l k} \otimes_{B} K
$$

Proof. By the standard constructions for tensor products and modules of differentials, it suffices to prove the lemma in the case where $K$ is a finitely generated field extension of $k$. Also, if $\omega \epsilon \Omega_{B / k}$ and $b \epsilon B$ are both non-zero but $b \omega=0$, then there is a finitely generated ring extension $O$ of $k[b]$ with quotient field $K$ such that the image of $\Omega_{C / k}$ in $\Omega_{B / k}$ contains 
$\omega$ and such that $b \omega^{\prime}=0$ for some preimage $\omega^{\prime}$ of $\omega$ in $\Omega_{O / k}$. By a theorem of Zariski ([25], p. 853), there is a regular spot $D$ containing $O$ and dominated by $B$. Clearly $\Omega_{D / k}$ has $D$-torsion. But this contradicts the fact that $\Omega_{D \mid k}$ is a free module ([17], p. 335).

2. Let $K$ be a field extension of a field $k$. If $B$ a valuation ring over $k$ with quotient field $K$, then by Lemma $1, \Omega_{B / k}$ can be identified with a submodule of $\Omega_{K / k}$. A differential in $\Omega_{K / k}$ is said to be $B$-regular if it is in $\Omega_{B / k}$ (identified with a submodule of $\Omega_{K / k}$ ), i.e. it is of the form $\sum a_{i} d b_{i}$ with $a_{i}, b_{i} \in B$. A differential in $\Omega_{K / k}$ is said to be of the first kind if it is $B$-regular for every valuation ring $B$ over $k$ with quotient field $K$. $A$ differential $\omega \epsilon \Omega_{K / k}$ is said to be of the second kind if for every such $B$ there is an $f \in K$ such that $\omega-d f$ is $B$-regular. Clearly every differential of the first kind is also of the second kind. For example, if $K$ is a function field of one variable over $k$, then a differential in $\Omega_{E / k}$ is of the first (resp. second) kind if and only if it is without poles (resp. residues) at all valuations of $K / k$.

If $\omega=\sum f_{i} d g_{i}$ is a differential in $\Omega_{K / k}$, then any derivation $D \in \operatorname{Der}_{k}(K, K)$ determines a differential

$$
(d \omega)(D, d)=\sum_{i}\left(D f_{i} d g_{i}-D g_{i} d f_{i}\right) .
$$

This is a differential in $\Omega_{K / k}$ which does not depend on the particular representation of $\omega$. If $D^{\prime}$ is another $k$-derivation of $K$, then $(d \omega)(D, \bar{d})$ aets on $D^{\prime}$ to yield

$$
(d \omega)\left(D, D^{\prime}\right)=\sum_{i}\left(D f_{i} D^{\prime} g_{i}-D g_{i} D^{\prime} f_{i}\right)
$$

The differential $\omega$ is said to be closed if $\langle d \omega\rangle(D, d)=0$ for all $D \in \operatorname{Der}_{k}(K, K)$.

If $K$ is a function field in one variable over $k$, then every differential $\omega \in \Omega_{K / k}$ is closed. If $K$ is the function field of an algebraic group defined over $k$ and $\omega$ (resp. $D_{1}$ and $D_{2}$ ) is a left invariant differential (resp. vector fields), then one can verify that ([16], p. 140)

$$
(d \omega)\left(D_{1}, D_{2}\right)=D_{1}\left(D_{2}^{*} \omega\right)-D_{2}\left(D_{1}^{*} \omega\right)-\left[D_{1}, D_{2}\right]^{*} \omega=-\left[D_{1}, D_{2}\right]^{*} \omega .
$$

In particular, if the group is commutative, then every left invariant differential is closed.

It will be useful to know that the various classes of differentials are stable under field extensions.

Limas 2. Let $k \subseteq K \subseteq L$ be a tower of fields and identify $\Omega_{K / k}$ with a $K$-submodule of $\Omega_{L / k}$.

(i) If $\omega \in \Omega_{\text {E/k }}$ is of the first kind (resp. second kind, closed) as a differential in $\Omega_{K / k}$, then it is also such as a differential in $\Omega_{L / k}$. (ii) Let $\bar{\omega}$ be the image of a differential $\omega$ by the canonical map $\Omega_{\text {Lf/k }}$ $\rightarrow Q_{L / K}$. If $\omega$ is of the first (resp. second) kind, then so is $\bar{w}$.

Proof. The assertion about elosed differentials is the only troublesome one. Clearly a differential $\omega \epsilon \Omega_{K / k}$ which is closed as an element of $\Omega_{L / k}$ is also closed as an element of $\Omega_{K / 2}$. Hence in proving the converse, it suffices to consider the case where $\mathbb{K} / k$ has a finite transcendence base $x_{1}, \ldots, x_{r}$. If $D \in \operatorname{Der}_{k}(L, L)$, then

$$
D=\sum_{i=1}^{r} D x_{i}\left(\frac{\partial}{\partial x_{i}}\right)+D^{\prime} \quad \text { with } \quad D^{\prime} \in \operatorname{Der}_{\bar{K}}(L, L) .
$$

It follows that

$$
(d \omega)(D, d)=\sum_{i=1}^{r} D x_{i}(d \omega)\left(\frac{\partial}{\partial x_{i}}, d\right)+(d \omega)\left(D^{\prime}, d\right) .
$$

Each term of the summation is zero since $\omega$ is closed, and if $\omega=\sum f_{i} d g_{i}$ with $f_{i}, g_{i} \in K$, then $(d \omega)\left(D^{\prime}, d\right)=0$ since $D^{\prime} f_{i}=D^{\prime} g_{i}=0$.

$A$ differential $\omega \in \Omega_{\text {K } / k}$ is called exact if it is of the form $\omega=d f$. If $E$ is a subset of $K$, we denote by $d E$ the set $\{d e \mid e \in E\}$. Every non-zero exact differential is of the second kind, but not of the first kind ([14], p. 169). A differential is called logarithmie if it is of the form df/f. A non-zero logarithmie differential is never of the second find ([14], p. 165).

If $\Delta$ is a set of $k$-derivations of a field $K$, then a differential $\omega \in \Omega_{K / k}$ is said to be $A$-exact if there is a $y \in K$ such that $D y=D^{*} \omega$ for all $D \in \Delta$. Any such $y$ will be denoted by an "indefinite integral" $y=\int_{A} \omega$.

3. The next lemma will not be used until section II.D.

LEMMA 3. Let $k \subseteq K \subseteq L$ be a tower of fields with $L$ algebraic over $K$ and $k$ algebraically closed in $L$, and suppose $\omega \in \Omega_{K / k}$. If $f_{\epsilon} L$ is such that $\omega-d f \in Q_{L / k}$ is a differential of the first hind, then $f \in K$.

Proof. It suffices to treat the case where ${ }^{\prime}$ is algebraically elosed and $L$ is a finite algebraic extension of $K$. Let $\sigma$ be any homomorphism of $L$ into the splitting field of $L / K$ such that $\left.\sigma\right|_{K}=i d_{K}$. By the universal mapping property of $\Omega_{L / k}$, the composite map

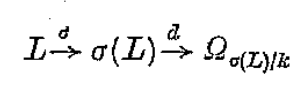

induces a map $\Omega_{L / k} \rightarrow \Omega_{\sigma(L) / k}$ such that $f d g \mapsto \sigma(f) d \sigma(g)$. This map admits an inverse induced by $\sigma^{-1}$, and it reduces to the identity map on $\Omega_{K / k}$. Since the isomorphism $\sigma$ establishes a bijection between the set of valuation rings of $L$ and those of $\sigma(L)$, the induced map takes differentials of the first (resp. second) kind to differentials of the same kind.

\footnotetext{
2- Acta Arithmetica $\mathrm{XXXII,2}$
} 
In particular, if $\omega-d f$ is of the first kind, then so is $\omega-d \sigma(f)$. Hence the difference $d(f-\sigma(f))$ is an exact differential of the second kind. But then ([14], p. 169), $d(f-\sigma(f))=0$, and so $f=\sigma(f)+a_{\sigma}$ with $a_{\sigma} \in k$. Summing over all $\sigma$, one gets

$$
\sum_{\sigma} f=\sum_{\sigma} \sigma(f)+\sum_{\sigma} a_{\sigma}=\operatorname{Tr}_{L / K}(f)+\sum_{\sigma} a_{\sigma} \in K
$$

which shows the lemma.

\section{B. The general theorem}

THeorem 1 . Let $F$ be a field of characteristic zero, $\Delta \subseteq \operatorname{Der}\left(F, F^{\prime}\right)$ be a set of $Q$-derivations of $F$, and $O=\bigcap_{D \in \Delta} \operatorname{ker} D$. Let $\omega_{1}, \ldots, \omega_{m}$ be closed $\Delta$-exact differentials of the second kind in $\Omega_{F / C} ; \omega_{1}^{\prime}, \ldots, \omega_{n}^{\prime}$ be closed differentials of the second kind in $\Omega_{F / C}$; and $\omega_{1}^{\prime \prime}, \ldots, \omega_{n}^{\prime \prime}$ be logarithmic differentials in $\Omega_{F / O}$ such that $D^{*} \omega_{i}^{\prime}=D^{*} \omega_{i}^{\prime \prime}$ for $D \in \Delta$ and $i=1, \ldots, n$. Suppose $E / C$ is a subextension of $F$ such that

(a) $\int_{\Delta} \omega_{i} \in E$ for $i=1, \ldots, m$,

(b) $\omega_{i}, \omega_{j}^{\prime}, \omega_{j}^{\prime \prime} \in \Omega_{E j C}$ for $i=1, \ldots, m ; j=1, \ldots, n$. If the following conditions are satisfied:

(i) $\omega_{1}, \ldots, \omega_{m}$ are C-linearly independent modulo $d E$ and

(ii) $\omega_{1}^{\prime \prime}, \ldots, \omega_{n}^{\prime \prime}$ are $Q$-linearly independent,

then tr.deg.o $E \geqslant m+n+r$ where $r$ is the dimension of the subspace of $F^{m+n}$ generated by the vectors $\left(D^{*} \omega_{1}, \ldots, D^{*} \omega_{m}, D^{*} \omega_{1}^{\prime \prime}, \ldots, D^{*} \omega_{n}^{\prime \prime}\right)$ as $D$ ranges through $\Delta$.

For ease of comparison, the notation of Ax [2] is preserved in the proof to as great a degree as possible.

Proof. (A) Change of coordinates. The first step is a change of coordinates analogous to ones found in proofis of the implicit function theorem ([8], p. 16). For notational convenience, set $\omega_{n+i}^{\prime \prime}=\omega_{i}$ for $i=1, \ldots, m$. There is a subset $J$ of $\{1,2, \ldots, m+n\}$ of cardinality $r$ and derivations $D_{j}$ for $j \in J$ such that $\operatorname{det}\left(D_{j}^{*} \omega_{i}^{\prime \prime}\right)_{J \times J} \neq 0$. Let $\left(a_{i j}\right)$ be the $r \times r$ matrix inverse of $\left(D_{i}^{*} \omega_{j}\right)$ and define for $i \in J$,

$$
D_{i}^{\prime}=\sum_{j \in J} a_{i j} D_{j} \in \operatorname{Der}(F, F)
$$

For each $D \in \Delta$, there are elements $b_{i}(D) \epsilon . F^{\prime}$ with

$$
D^{*} \omega_{j}^{\prime \prime}=\sum_{i \in J} b_{j}(D) D_{i}^{*}\left(\omega_{j}^{\prime \prime}\right) \quad \text { for } j \in J .
$$

Define $D^{\prime}=D-\sum_{i \in J} b_{i}(D) D_{i} \in \operatorname{Der}\left(\dot{F}, F^{\prime}\right)$ and set

$$
\Delta^{\prime}=\left\{D_{i}^{\prime} \mid i \epsilon J\right\} \cup\left\{D^{\prime} \mid D \in \Delta\right\} \text {. }
$$

LEMMA. In addition to all the conditions on $\Delta$, the set $\Delta^{\prime}$ also satisfies:

$$
\left\{\begin{array}{l}
D_{i}^{\prime *} \omega_{j}^{\prime \prime}=\delta_{i j} \text { (Fronecter } \delta \text { ) for } i, j \epsilon J \text { and } \\
D^{\prime *} \omega_{j}^{\prime \prime}=0 \text { for } j \epsilon J \text { and } D \neq D_{j} .
\end{array}\right.
$$

Proof. The fact that $\Delta^{\prime}$ satisfies all the conditions on $\Delta$ follows immediately from the definitions and the observation that $\Delta$ and $\Delta^{\prime}$ generate the same $F$-linear subspace of $\operatorname{Der}\left(F, F^{\prime}\right)$. Condition (1) follows from the definitions of $\left(a_{i j}\right)$ and the $b_{i}(D)$.

In view of the lemma, we remove the primes from the $D$ 's of (1) and suppose it to hold for 4 .

(B) Construction of a differential relation. Suppose that tr.deg. $E<m+n+r$. Let $\omega_{n+i}^{\prime}=d_{F / C}\left(\int \omega_{i}\right)$ for $i=1, \ldots, m$. Then the $m+n+r$ differentials

$$
\omega_{i}^{\prime}-\omega_{i}^{\prime \prime}, \omega_{j}^{\prime \prime} \quad \text { for } \quad i=1, \ldots, m \div n ; \quad j \in J
$$

are $E$-linearly dependent and a fortiori $F$-linearly dependent. Let

$$
\sum_{i=1}^{m+n} \alpha_{i}\left(\omega_{i}^{\prime}-\omega_{i}^{\prime \prime}\right)+\sum_{j \in J} \beta_{j} \omega_{j}^{\prime \prime}=0
$$

be an equation of $\mathbb{F}$-linear dependence with the least possible positive number of non-zero coefficients. By dividing through by one of the nonzero coefficients, we can require that at least one of the $\alpha_{i}$ or $\beta_{j}$ be equal to 1.

(C) Triviality of the $\beta_{j}$. For any derivation $D \in \operatorname{Der}_{C}(F, F)$ one can define

$$
D^{\mathrm{1}}: Q_{F / C} \rightarrow \Omega_{F / C} \quad \text { by } \quad f d g \mapsto D f d g+f d(D g)
$$

and $C$-linearity. This map satisfies the Leibnitz rule:

$$
D^{1}(f \omega)=f D^{x} \omega+(D f) \omega \text {. }
$$

LEMMa. If $\omega \in \Omega_{E / C}$ is a differential and $D \in \operatorname{Der}_{\sigma}(F, F)$ is a derivation with $d\left(D^{*} \omega\right)=0$ and $(d \omega)(D, d)=0$, then $D^{1} \omega=0$.

Proof. Suppose $\omega=\sum_{i} u_{i} d v_{i}$. Then.

$$
d\left(D^{*} \omega\right)=d\left(\sum_{i} u_{i} D v_{i}\right)=\sum_{i}\left(d u_{i} D v_{i}+u_{i} d D v_{i}\right)
$$

$\operatorname{and}$

$$
(d \omega)(D, d)=\sum_{i}\left(D u_{i} d v_{i}-d u_{i} D v_{i}\right)
$$


Hence

$$
\begin{aligned}
D^{1} \omega & =\sum_{i}\left(D u_{i} d v_{i}+u_{i} d D v_{i}\right) \\
& =\left(\sum_{i} D u_{i} d v_{i}-d u_{i} D v_{i}\right)+\left(\sum_{i} d u_{i} D v_{i}+u_{i} d D v_{i}\right) \\
& =(d \omega)(D, d)+d\left(D^{*} \omega\right)=0 .
\end{aligned}
$$

By hypothesis, the $\omega_{i}^{\prime}-\omega_{i}^{\prime \prime}$ are closed and $D^{*}\left(\omega_{i}^{\prime}-\omega_{i}^{\prime \prime}\right)=0$ for all $D \in \Delta$ and $i=1, \ldots, m+n$. Further the $\omega_{j}^{\prime \prime}$ are closed and $d D^{*} \omega_{j}^{\prime \prime}=0$ for $j \in J$ by part (A) of the proof. Applying the lemma gives

(3) $D^{\mathrm{1}}\left(\omega_{i}^{\prime}-\omega_{i}^{\prime \prime}\right)=D^{1} \omega_{j}^{\prime \prime}=0 \quad$ for $\quad i=1, \ldots, m+n ; \quad j \in J, D \in \Delta$.

Applying $D^{1}$ to $(2)$ and simplifying with the relations (3) shows

$$
\sum_{i=1}^{m+n} D \alpha_{i}\left(\omega_{i}^{\prime}-\omega_{i}^{\prime \prime}\right)+\sum_{j \in J} D \beta_{j} \omega_{j}^{\prime \prime}=0
$$

for all $D \in \Delta$. Since at least one of the $\alpha_{i}$ or $\beta_{j}$ was taken to be 1 , this is an equation of $F$-linear dependence with fewer non-zero coefficients than in the minimal equation (2). By minimality $D \alpha_{i}=D \beta_{j}=0$ for $i=1, \ldots$ $\ldots, m+n ; j \in J$, and $D \in \Delta$. Since $O=\bigcap \operatorname{ker} D$, it follows that $a_{i}, \beta_{j} \in C$ are constants. Furthermore, applying $D_{j}^{*}$ to (2) and using (1), it follows that $\beta_{j}=0$ for all $j \in J$.

(D) The residue argument. Let $z_{i} \epsilon F$ for $i=1, \ldots, n$ be such that $\omega_{i}^{\prime \prime}=d z_{i} / z_{i}$ and let $\gamma_{1}, \ldots, \gamma_{s}$ be a basis for the $Q$-linear subspace of $O$ generated by $\alpha_{1}, \ldots, \alpha_{n}$. Suppose $0 \neq q$ and $n_{i j}$ are integers chosen so that

$$
q \alpha_{i}=\sum_{j=1}^{s} n_{i j} \gamma_{j} \quad \text { for } \quad i=1, \ldots, n
$$

and set

$$
v_{j}=\prod_{i=1}^{n} z_{i}^{n_{i j}}
$$

Then one can verify that

$$
\sum_{i=1}^{n} \alpha_{i}\left(\frac{d z_{i}}{z_{i}}\right)=(1 / q) \sum_{j=1}^{s} \gamma_{j}\left(\frac{d v_{j}}{v_{j}}\right)
$$

By hypothesis, $\omega_{i}^{\prime \prime}, \omega_{i}^{\prime}, \omega_{j}$ for $i=n+1, \ldots, n+m ; j=1, \ldots, n$ are differentials of the second kind. Let $K / O$ be a function field containing all of the $z_{i}$ and satisfying the definition of differential of the second kind as applied to these $2 m+n$ differentials. Suppose $d v_{j} \neq 0$ for at least one $j \leqslant n$. Let $E^{\prime} / C$ be a subfield of $K$ algebraically closed in $K$ with tr. deg. $\cdot_{E^{\prime}} K=1$ and $v_{j}$ transcendental over $E^{\prime}$ for some $j \leqslant n$. Applying the canonical map $\Omega_{K / C} \rightarrow Q_{K / E}$ to equation (2) yields a relation of the same form denoted $\left(2^{\prime}\right)$, and this relation can be viewed as occurring in $Q_{\bar{K} \bar{E}^{\prime} / \bar{E}^{\prime}}$ where $\bar{E}^{\prime}$ is an algebraic closure of $E^{\prime}$.

If $p$ is any place of $\bar{\hbar} \bar{E}^{\prime}$ over $\bar{E}^{\prime}$, then taking residues ([14], p. 165) in $(2 ')$ yields in view of (4) that

$$
(-1 / q) \sum_{j=1}^{s} \gamma_{j} \operatorname{Res}_{p}\left(\frac{d v_{j}}{v_{j}}\right)=0 \quad \text { for all } p \text {. }
$$

But $\operatorname{Res}_{p}\left(\frac{d v_{j}}{v_{j}}\right)=\operatorname{ord}_{p} v_{j} \in Z$ ([14], p. 169). Since the $\gamma_{j}$ are $Q$-linearly independent, this last equation gires ord $v_{j}=0$ for all p. Hence $v_{j} \in \bar{E}^{\prime} \cap K$ $=E^{\prime}$ contrary to hypothesis. Thus $d v_{j}=0$ for all $j=1, \ldots, s$.

Differentiating $v_{j}=\prod_{i=1}^{n} z_{i}^{n_{i j}}$ rields

$$
0=\left(\frac{d v_{j}}{v_{j}}\right)=\sum_{i} n_{i j}\left(\frac{d z_{i}}{z_{i}}\right)=\sum_{i=1}^{n} n_{i j} \omega_{i}^{\prime \prime}
$$

By hypothesis (ii) of the theorem, $n_{i j}=0$ for all $i$ and $j$, and so $\alpha_{i}=0$ for $i=1, \ldots, n$. Since $a_{i}=\beta_{j}=0$ for $i=1, \ldots, n ; j \in J,(2)$ is an equation of $C$-linear dependence of $\omega_{1}, \ldots, \omega_{m}$ and $d_{F / C}\left(\sum_{i=1}^{n} a_{n+i} \int_{4} \omega_{i}\right)$. By hypotheses (a) and (i) of the theorem, it follows that $\alpha_{n+i}=0$ for $i=1, \ldots$ $\ldots, m$. This contradicts our choice of $(2)$, and so the theorem is proved.

COROITARY 1. (a) The conclusion of the theorem still holds true when condition (i) is replaced by either of the following conditions:

1) $\omega_{1}-d \int_{\Delta} \omega_{1}, \ldots, \omega_{m}-d \int_{4} \omega_{m}$ are C-linearly independent.

2) $\omega_{1}, \ldots, \omega_{n}$ are differentials of the jirst kind and either $\int_{\Delta} \omega_{1}, \ldots, \int_{\Delta} \omega_{m}$ are C-linearly independent modulo $O$ or else $\omega_{1}, \ldots, \omega_{m}$ are $C$-linearly independent.

(b) If $n=0$, the conclusion of the theorem still holds true when one deletes the condition that $\omega_{1}, \ldots, \omega_{m}$ be of the second kind.

Proof. (A) Suppose condition 1) holds but that $\sum_{i=1}^{m} \alpha_{i} \omega_{i}=d u$ with $\alpha_{i} \epsilon \dot{C}$ and $u \in F$. For $D \in \Delta$, apply $D^{*}$ to the following equation

$$
\sum_{i=1}^{m} \alpha_{i}\left(\omega_{i}-d \int_{\Delta} \omega_{i}\right)=d\left(u-\sum_{i=1}^{m} \alpha_{i} \int_{\Delta} \omega_{i}\right)
$$


to conclude that

$$
0=D\left(u-\sum_{i=1}^{m} \alpha_{i} \int_{\Delta} \omega_{i}\right) \quad \text { for all } D \in \Delta .
$$

Since $\bigcap_{D \in A} \operatorname{ker} D=C$, it follows that $u-\sum_{i=1}^{m} \alpha_{i} \int_{\Delta} \omega_{i} \epsilon C$, and so $d u=\sum \alpha_{i} d \int_{\Delta} \omega_{i}$. Thus

$$
\sum_{i=1}^{m} a_{i}\left(\omega_{i}-d \int_{\Delta} \omega_{i}\right)=0
$$

and so $\alpha_{i}=0$ for $i=1, \ldots, n$ by condition 1). Hence condition (i) of the theorem holds, and the conclusion follows.

(B) Condition 2) implies condition 1). In fact, suppose

$$
\sum_{i=1}^{m} \alpha_{i} \omega_{i}=d\left(\sum_{i=1}^{m} \alpha_{i} \int_{\Delta} \omega_{i}\right)
$$

By condition 2), the left hand side is a differential of the first kind. The right hand side is exact and hence not of the first kind unless it is zero. Hence

$$
\sum_{i=1}^{m} \alpha_{i} \omega_{i}=0 \quad \text { and } \quad \sum_{i=1}^{m} \alpha_{i} \int_{\Delta} \omega_{i} \epsilon C .
$$

By condition 2), $a_{i}=0$ for $i=1, \ldots, m$ as desired.

(C) The condition that $\omega_{1}, \ldots, \omega_{m}$ be of the second kind was used only in the residue argument. If $n=0$, this part of the argument may be omitted.

C. Applications to Weierstrass $\wp$-functions. For simplicity, we restrict ourselves to the one variable case.

COROXXARY 2. For $i=1, \ldots, n$, let $\wp_{i}(z)$ be a Weierstrass. $\wp$-function with invariants $g_{i 2}, g_{i 3}$ respectively, and let $y_{i}(z)$ be a function holomorphio in a neighborhood of a point $a_{\epsilon} \boldsymbol{C}$ and vanishing at a. Suppose $y_{1}, \ldots, y_{n}$ are linearly independent over a field $K$ which contains all the invariants of the $\wp_{i}$ as well as the derivatives $y_{i}^{(m)}(a)$ of all orders $m>0$. Then

tr. $\operatorname{deg} \cdot \boldsymbol{C} C\left(y_{1}(z), \ldots, y_{n}(z), \wp_{1}\left(y_{1}(z)\right), \ldots, \wp_{n}\left(y_{n}(z)\right)\right) \geqslant n+1$.

CoRoLIARY 3. Under the hypotheses of Corollary 2,

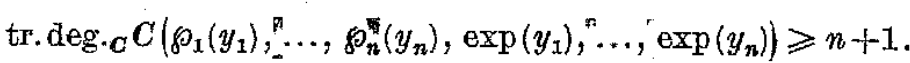

COROLLARX 4. Under the hypotheses of Corollary 2 , if $\beta_{1}, \ldots, \beta_{n} \in K^{*}$, then the field generated over $\boldsymbol{C}$ by all the $\dot{y}_{i}{ }^{\prime} s^{\prime}, \wp_{i}\left(y_{i}\right) ' s$, and $\exp \left(\beta_{i} y_{i}\right)^{\prime} s$ is of transcendence degree at least $2 n+1$ over $C$.
COROLLARY $5(\mathrm{Ax})$. If $y_{1}, \ldots, y_{n}$ are Q-linearly independent power series without constant terms, then

$$
\text { tr. deg. } \boldsymbol{C} C\left(y_{1}, \ldots, y_{n}, \exp \left(y_{1}\right), \ldots, \exp \left(y_{n}\right)\right) \geqslant n+1 .
$$
Then

COROLAARY 6. If $\wp$ is a Weierstrass $\wp$-function, let $T(z)=1 / \wp(z)$.

$$
\text { tx.deg.c } C(z, T(z), T \circ T(z), \ldots, \underbrace{T \circ T \circ \ldots \circ T(z)}_{n \text {-times }})=n+1 .
$$

Proof (of corollaries). Identifying the $y_{i}$ with their power series expansions about $a$, one can suppose $y_{i} \in K[[u], u=z-a$. Let $F$ be the field of formal Laurent series in $u$ over $c, \Delta=\left\{\frac{\partial}{\partial u}\right\}$ so that $\sigma=\bigcap_{D \in \Delta} \operatorname{ker} D=C$. Note that since the $y_{i}$ have no constant terms, they are $\mathcal{K}$-linearly independent if and only if they are linearly independent over $K$ modulo $K$. Since $K$ contains all the coefficients of the $y_{i}(u)$, this in turn implies that they are $\boldsymbol{C}$-linearly independent modulo $\boldsymbol{C}$.

The function field $C\left(\wp_{i}(z), \wp_{i}^{\prime}(z)\right)$ over $C$ of the elliptic curve

$$
\left(\wp_{i}^{\prime}(z)\right)^{2}=4\left(\wp_{i}(z)\right)^{3}-g_{i 2} \wp_{i}(z)-g_{i 3}
$$

has a differential of the first kind $\frac{d \wp_{i}(z)}{\wp_{i}^{\prime}(z)}$ ([14], p. 163). Since $\wp_{i}\left(y_{i}\right)$ is non-constant and hence transcendental over $C, \omega_{i}=\frac{d \wp_{i}\left(y_{i}\right)}{\wp_{i}^{\prime}\left(y_{i}\right)}$ is a differential of the first kind of the function field $C\left(\wp_{i}\left(y_{i}\right), \wp_{i}^{\prime}\left(y_{i}\right)\right)$. By Lemma $2, \omega_{i}$ is a differential of the first kind in $\Omega_{F / \sigma}$. Clearly $\omega_{i}$ is $\Delta$-exact with $\int_{d} \omega_{i}=y_{i}$.

Similarly one has logarithmic differentials $e^{-\beta_{i} y_{i}} d e^{\beta_{i} v_{i}}=\omega_{i}^{\prime \prime}$ which are $\Delta$-exact with $\int \omega_{i}^{\prime \prime}=y_{i}$. In view of these remarks, Corollary 4 and hence also Corollaries 2 and 3 are consequences of Corollary 1 . The proof of Corollary 5 is similar, but using only logarithmie differentials. Finally Corollary 6 follows by an induction on $n$ using $\wp_{1}=\wp_{2}=\ldots=\wp_{n}=\wp_{2}$ and $y_{1}=z, y_{i+1}=T\left(y_{i}\right)$ for $i=1, \ldots, n-1$.

\section{GEOMETRIC INTERPRETATION}

A. Algebraie varieties. Let $P$ be a $l a$ rational point on a non-singular irreducible projective algebraic variety $V$ of dimension $n$ defined over a field $k$ of eharacteristic zero. Mapping a set of uniformizing parameters 
of $V$ at $P$ to the indeterminants $x_{1}, \ldots, x_{n}$ defines a local homomorphism

$$
o_{P}(V) \rightarrow k\left[\left[x_{1}, \ldots, x_{n}\right]\right]
$$

of the local ring of $P$ on $V$ into the power series ring in $n$ variables. Let $k^{\prime}$ be a field extension of $k$ which is algebraically free from $k\left(x_{1}, \ldots, x_{n}\right)$ over $k$. If $y_{1}, \ldots, y_{n}$ are power series in $k^{\prime}\left[\left[t_{1}, \ldots, t_{s}\right]\right]$ without constant terms, then one has a local homomorphism defined by mapping $x_{i}$ to $y_{i}$ for atl $i$. If $p$ is the kernel of the composition of the two maps, then the subrariety $T$ of $V$ defined by $p$ is called the k-subrariety defined by $f_{1}, \ldots, y_{n}$. Clearly $W$ depends on the choice of uniformizing parameters.

A differential $\omega \in \Omega_{k(V) / k}$, where $k(V)$ is the function field of $V$, is said to be regular at $P$ if it is expressible in the form $\omega=\sum_{i=1}^{n} u_{i} d v_{i}$ with $u_{i}, v_{i} \in O_{P}(V)$. If $\omega$ is of this form, then it induces a differential $\omega_{W} \epsilon \Omega_{k(F) / h}$ defined by reducing the $u_{i}$ and $v_{i}$ modulo $p$ ([9]). The differential $\omega_{W}$ can be viewed as a differential in $\Omega_{k^{\prime}((t)) / k^{\prime}}$.

Now apply Corollary 1 with $f=k^{\prime}\left(\left(t_{1}, \ldots, t_{\mathrm{s}}\right)\right), \quad C=k^{\prime}$, and $\Delta=\left\{\left(\frac{\partial}{\partial t_{j}}\right) \mid j=1, \ldots, s\right\}$. One gets the following result.

COROLLARY 7. With the above notation, let $\omega_{1}, \ldots, \omega_{n}$ be a set of $k$ linearly independent closed $\Delta$-exact differentiats of the first kind on $V$, all regular at $P$. Let $z_{1}, \ldots, z_{m}$ be $Q$-linearly independent power series in $k^{\prime}\left[\left[t_{1}, \ldots, t_{s}\right]\right]$ and suppose $z_{i}(0, \ldots, 0)=0$ for all $i$. Suppose further that either the $k$-subvariety. defined by $y_{1}, \ldots, y_{n}$ is either $V$ itself or else that $\int_{d}\left(\omega_{1}\right)_{W}, \ldots, \int_{4}\left(\omega_{n}\right)_{W}$ are $k^{\prime}$-linearty independent modulo $k^{\prime}$.

$$
\text { tr. deg. } \cdot^{\prime} k^{\prime}\left(y_{1}, \ldots, y_{n}, \int_{\Delta}\left(\omega_{1}\right)_{W}, \ldots, \int_{\Delta}\left(\omega_{n}\right)_{W}, z_{1}, \ldots, z_{m}, e^{z_{1}}, \ldots, e^{z_{m}}\right)
$$

is at least $n+m+r$ where $r$ is the dimension over $k^{\prime}\left(\left(t_{1}, \ldots, t_{s}\right)\right)$ of the vector space generated by the vectors

$$
\left(\left(\frac{\partial}{\partial t_{j}}\right)^{*}\left(\omega_{1}\right)_{W}, \ldots,\left(\frac{\partial}{\partial t_{j}}\right)^{*}\left(\omega_{n}\right)_{W}, \frac{\partial z_{1}}{\partial t_{j}}, \ldots, \frac{\partial z_{m}}{\partial t_{j}}\right)
$$

yor $j=1, \ldots, s$.

B. Algebraic curves. Let $O$ be a nonsingular complex projective plane curve passing through the origin. A Puiseux expansion about the origin ([14]; p. 104) gives a parametrization $:=T^{k}, y=q(T)$. Let $R(x, y) d x$ be a differential of the first kind on $O$, and let $L_{R}$ be a formal Laurent series solution of

$$
\left(d L_{R} / d T\right)=k T^{k-1} R\left(T^{k}, q(T)\right) .
$$

The result of part $A$ gives
COROLXARY 8. Let $y_{1}, \ldots, y_{n}, z_{1}, \ldots, z_{m} \epsilon C\left[\left[t_{1}, \ldots, t_{s}\right]\right]$ be formal power series without constant terms. Suppose $z_{1}, \ldots, z_{m}$ are Q-linearly independent and $1, L_{R}\left(y_{1}\right), \ldots, L_{R}\left(y_{n}\right)$ are C-linearly independent. Then

$$
\text { tr.deg.c } C\left(y_{1}, \ldots, y_{n}, L_{R}\left(y_{1}\right), \ldots, L_{\mathcal{R}}\left(y_{n}\right), z_{1}, \ldots, z_{m}, e^{z_{1}}, \ldots, e^{z_{m}}\right)
$$

is at least $n+m+r$, where $r$ is the dimension of the vector space spanned by $\left(\frac{\partial y_{1}}{\partial t_{j}}, \ldots, \frac{\partial y_{n}}{\partial t_{j}}, \frac{\partial z_{1}}{\partial t_{j}}, \ldots, \frac{\partial z_{m}}{\partial t_{j}}\right)$ for $j=1, \ldots, s$.

C. Algebraic groups. Let $G$ be a connected commutative complex algebraic group with identity $e, \mathscr{G}=T_{e} G$ be its Lie algebra, exp: $\mathscr{G} \rightarrow G$ the exponential map, $o_{e}$ the local ring of rational functions regular at $e$, and $O_{e}$ the ring of germs of analytic functions at $e$. The ring of germs of analytic functions of $\mathscr{G}$ at the origin is of the form $C\left\langle\langle x\rangle=C\left\langle x_{1}, \ldots\right.\right.$ $\left.\ldots, x_{n}\right\rangle, n=\operatorname{dim} G$. Choose $y_{1}, \ldots, y_{n}$ in $k\left[\left[t_{1}, \ldots, t_{r}\right]\right]$ to be formal power series without constant terms. If one denotes by $(\exp )_{\epsilon}^{*}: T_{e}^{*} G \rightarrow T_{e}^{*} \mathscr{G}$ $([16], p .145)$ the map of the cotangent spaces induced by the exponential map, then one can form the commutative diagram:

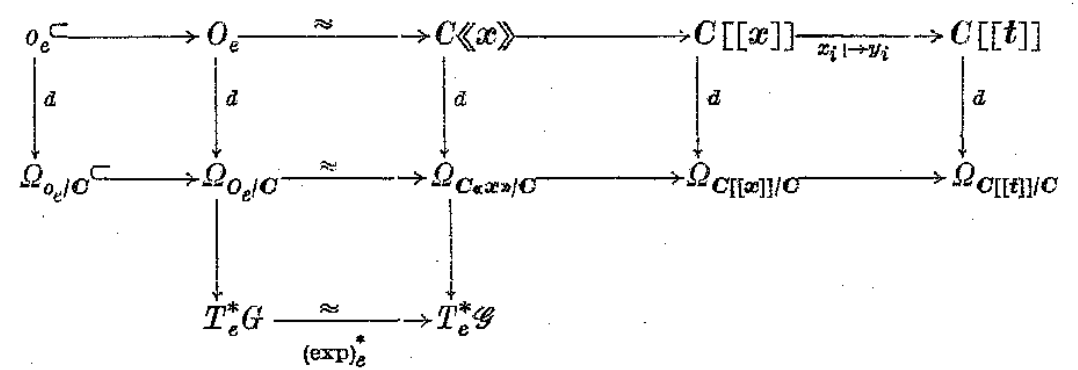

where the maps are either the obvious ones or those obtained from the universal property of the differentials.

The $\boldsymbol{C}$-rector space of left invariant differentials of $G([21], p .9)$, has a basis $\omega_{1}, \ldots, \omega_{n} \in \Omega_{o_{\ell} / \boldsymbol{C}}$. These map via the diagram to a basis for the left invariant differentials of the Lie group $G$ as they are represented in $T_{e}^{*} G$. The left invariant differentials of $\mathscr{G}$ are represented in $T_{e}^{*} \mathscr{G}$ by the subspace generated by the images of the $d x_{i}$ for $i=1, \ldots, n$. Let $\Delta_{1}$ denote the set of partial derivatives $\partial / \partial x_{i}$ for $i=1, \ldots, n$. The map $(\exp )_{e}^{*}$ identifies the left invariant differentials and so the images of the $\omega_{i}$ in $\Omega_{C\langle\langle x\rangle\rangle / C}$ are $\Delta_{1}$-exact with $\int^{\prime} \omega_{i}$ equal to a linear combination of the $x_{j}{ }^{\prime}$ s. It follows that the images $\bar{\omega}_{i}$ in $\Omega_{C[f]] / O}$ of the $\omega_{i}$ 's are $\Delta$-exact where $\Delta=\left\{\frac{\partial}{\partial t_{j}} \mid j=1, \ldots, r\right\}$ and that $\int_{\Delta} \bar{\omega}_{i}$ is a linear combination of the $y_{j}$ 's. Since $G$ is commutative, the $\omega_{i}$ 's are closed ( $\$ \mathrm{I}, \mathrm{A} .2$ ).

Now suppose $G=L \times A$ where $A$ is an Abelian variety and $L \approx\left(G_{m}\right)^{t}$ is isomorphic to a product of multiplicative groups $G_{m}$, i.e. $L$ is a com- 
mutative linear group with no unipotent part ([21], pp. 4-12). Suppose the notation above is chosen so $y_{1}, \ldots, y_{t}$ correspond to $L$ and $y_{i+1}, \ldots, y_{n}$ correspond to $A$. The exponential map of $L$ is $\left(u_{1}, \ldots, u_{t}\right) \mapsto\left(e^{u_{1}}, \ldots, e^{u_{t}}\right)$, and $L$ has its left invariant differentials generated by $d x_{i} / x_{i}$. The algebraic subgroups of $L$ correspond to $Q$-linear subspaces of the Lie algebra $C^{t}$ of $L$. The exponential map of $A$ identifies $A$ with $C^{n-t} / \Lambda$ where $C^{n-t}$ is the Lie algebra of $A$ and $A$ is a full lattice in $C^{n-t}$ ([22], p. 21). The algebraic subgroups of $A$ correspond to subspaces $V$ of $C^{n-t}$ containing sublattices of $A$ of dimension twice that of $\nabla$ ([21], p. 21).

We wish to apply Corollary 1 to the differentials of the first kind $\bar{\omega}_{i+1}, \ldots, \bar{\omega}_{n} \in Q_{\boldsymbol{C}[\boldsymbol{t}]] / \boldsymbol{C}}$ and the logarithmic differentials $\bar{\omega}_{i}=e^{-y_{i}} d e^{y_{i}}$, $i=1, \ldots, t$. If one could verify the hypotheses of the corollary, then for any field $E$ containing the $y_{i}$ 's and the field of fractions $\bar{K}$ of the image $\vec{o}_{e}$ in $\boldsymbol{C}((\boldsymbol{t}))$ of $o_{e}$ would have transcendence degree $\geqslant n+r$ where $r$ is the rank of the matrix $\left(\partial y_{i} / \partial t_{j}\right)$. To guarantee the hypothesis of the corollary, the next lemma is used.

LEMuA 4. If $y_{t+1}, \ldots, y_{n}$ do not lie in any proper subspace $\nabla$ of $C^{n-t}$ containing a sublattice of $\Lambda$ of dimension twice that of $\nabla$, then $\bar{\omega}_{t+1}, \ldots, \bar{\omega}_{n}$ are $\boldsymbol{C}$-linearly independent.

Proof. Let $W$ be the subvariety of $A$ defined by $y_{t+1}, \ldots, y_{n}$, and suppose $\omega=\sum_{i m t+1}^{n} \alpha_{i} \omega_{i}$ is a non-trivial linear combination with $\omega_{F}=\sum_{i=t+1}^{n} a_{i} \bar{\omega}_{i}=0$. Since the $\omega_{i}$ are closed, $\omega=0$ determines a completeIy integrable differential system ([16], p. 159), $\left\{\mathfrak{S}_{g}\right\}, \mathfrak{H}_{g}=\left\{X_{g} \mid X \in \mathrm{ker}^{*} \omega\right\}$ on the Lie group $A$. Since $A$ is commutative, ker $\omega$ is a Lie subalgebra of $G$. Hence $W$ is contained in the Lie subgroup of $A$ corresponding to ker $\omega$. Since $\omega \neq 0$, this Lie subgroup is proper. But then the group generated by $W$ is proper. Since $W$ is irreducible, Zariski closed, and contain. the identity of $\mathcal{A}$, the group generated by $W$ is an algebraic subgroup of $A$ ([21], p. 143), and so is a proper algebraic subgroup of $A$. By the correspondence of the last paragraph, $y_{t+1}, \ldots, y_{n}$ lie in a subspace $V$ of $C^{n-t}$ containing a sublattice of $A$ of dimension twice that of $V$.

Applying the lemma, one sees by Corollary 1:

ConollaRX 9. With the notation as above, suppose that $y_{1}, \ldots, y_{i}$ are $Q$-linearly independent and that $y_{t+1}, \ldots, y_{n}$ do not lie in a subspace $V$ of $C^{n-t}$ containing a sublattice of $\Lambda$ of dimension twice that of $V$. Then any subfield $E$ of $C((t))$ with $E \bar{K}\left(y_{1}, \ldots, y_{n}\right)$ algebraic over $B$ is of transcendence degree over $\boldsymbol{C}$ at least $n+r$ where $r$ is the rank of the Jacobian matrix $\left(\partial y_{i} / \partial t_{j}\right)$.

D. Weierstrass functions. he considerations of the last section lead to necessary and sufficient conditions for the algebraic independence of Weierstrass elliptic functions and exponential functions.
We begin by studying products of lattices. Let $\pi_{1}, \pi_{1}^{\prime}, \ldots, \pi_{n}, \pi_{n}^{\prime}$ be non-zero complex numbers such that the ratios $\tau_{i}=\pi_{i}^{\prime} / \pi_{i}$ satisfy the following conditions:

(a) $\tau_{i}$ is not real for $i=1, \ldots, n$,

(b) $\tau_{i} \neq \frac{a \tau_{j}+b}{e \tau_{j}+d}$ whenever $i \neq j$ and $a, b, c, d \epsilon \boldsymbol{Z}$ with $a d-b c \neq 0$.

Define for $i=1, \ldots, n$,

$$
K_{i}= \begin{cases}Q\left(\tau_{i}\right) & \text { if } \tau_{i} \text { is quadratic, } \\ Q & \text { otherwise. }\end{cases}
$$

For the same range of $i$, let $\lambda_{i}$ be the lattice

$$
\lambda_{i}=\boldsymbol{Z} \pi_{i}+\boldsymbol{Z} \pi_{i}^{\prime}=\left\{n_{1} \pi_{i}+n_{2} \pi_{i}^{\prime} \mid n_{1}, n_{2} \in \mathbf{Z}\right\} \text {. }
$$

Let $m_{1}, \ldots, m_{n}$ be positive integers, $d=m_{1}+\ldots+m_{n}$, and define the internal direct sums for $i=1, \ldots, n$ :

$$
\begin{aligned}
\Lambda_{i} & =\lambda_{i}^{m_{i}} \subseteq C_{i}^{m_{i}}=W_{i}, \\
A & =A_{1} \times \ldots \times A_{n} \subseteq C^{d} .
\end{aligned}
$$

Then $A$ is a discrete $2 d$-dimensional lattice in $C^{d}$.

Let $L$ be a $k$-dimensional $C$-linear subspace of $C^{d}$. It must have dimension $2 k$ as an $\boldsymbol{R}$-vector space. Since $A$ is discrete, it follows from Kronecker's Theorem ([9], p. 382), that the dimension of the lattice In $\Lambda$ is at most $2 k$. When it is exactly $2 k$, the following crucial result shows that $L$ must be of a very special form indeed.

Propositron. If $L$ is a k-dimensional $C$-linear subspace of $C^{d}$ whioh contains a 2k-dimensional sublattice of $A$, then $L$ has a basis over $C$, each element of which lies in some $W_{i}$ and has coordinates in $K_{i}$.

Proof. The proof is by induction on $d$ with $d=1$ being a trivial case. Assuming $d>1$, the proof falls naturally into two not entirely disjoint cases:

Case 1 . There is an element $v \in L \cap W_{1}$ with all $m_{1}$ coordinates from $K_{1}$ and the first coordinate non-zero. The subset of $l$ consisting of all elements of $L$ with zero first coordinate can be considered to be a subspace of $C^{d-1}$ of dimension $k-1$. As such it is a sublattice of $\Lambda^{m_{1}-1} \times \Lambda_{2} \times$ $\times \ldots \times A_{n}$ of dimension $2(k-1)$. In fact, if $K_{1}=Q$, then clearing the denominators of $v$ with some integer $t$ gives two $Z$-linearly independent elements $t \pi_{1} v$ and $t \pi_{1}^{\prime} v$ in $\Lambda$. If $K_{1}=Q\left(\tau_{1}\right)$, the vector $\tau_{1} v$ has coordinates of the form $a+b \tau_{1}$ with $a, b \in Q$; so for some $t \in Z$, the vectors $t \pi_{1} v$ and $t \pi_{1}^{\prime} v=t \pi_{1}\left(\tau_{1} v\right)$ of $\Lambda$ are $Z$-linearly independent. In either case, for every $\lambda \in t \Lambda$, there are integers $c$ and $d$ such that the first coordinate of $\lambda-c t \pi_{1} v-d t \pi_{1}^{\prime} v$ is zero. Hence $L \cap\left(\lambda_{1}^{m_{1}-1} \times \Lambda_{2} \times \ldots \times \Lambda_{n}\right)$ has dimension at least $2(k-1)$. Since $L \cap C^{a-1}$ has dimension $k-1$ and $A$ is discrete, the dimension of the sublattice is exactly $2(k-1)$. 
By induction, this subspace $L \cap C^{d-1}$ has a basis $B$ consisting of vectors each lying in some $W_{i}$ and having coordinates from $K_{i}$, and $B \cup\{v\}$ is a basis of $L$ of the same kind. In particular, this case applies when $L$ contains a non-zero element whose only non-zero coordinate is in the first place.

Case 2. Zero is the only element of $L$ whose final $d-1$ coordinates are zero. Then the projection $p: C^{d} \rightarrow C^{d-1}$ onto the final $d-1$ coordinates is injective when restricted to $L$. Now $p(L)$ is a $k$-dimensional $C$-subspace of $C^{d-1}$ containing a sublatice of $A$ of dimension $2 k$. By induction, $p(L)$ has a basis $B^{\prime}$ of the desired type. If each $b^{\prime} \in B^{\prime}$ is of the form $p(b)$ for some $b \in L$ with first coordinate zero, then these $b$ 's form a. basis of $L$ of the type needed.

At any rate, since the dimension of $p(L) \cap A$ is at most 27, and 27 is the dimension of $p(L \cap A)$, the $Q$-vector space $\nabla$ spanned by $L \cap A$ projects br $p$ onto the $Q$-vector space spanned by $p(L) \cap A$. We know that each $b^{\prime} \in B^{\prime}$ is in $W_{i}$ for some $i$. For such an $i, \pi_{i} b^{\prime}$ and $\pi_{i}^{\prime} b^{\prime}$ both lie in $W_{i}$ and in the $Q$-vector space spanned by $p(L) \cap A$. Hence there are elements $b^{*}$ and $b^{* *}$ in $V$ with $p\left(b^{*}\right)=\pi_{i} b^{\prime}, p\left(b^{* *}\right)=\pi_{i}^{\prime} b^{\prime}$ and whose first coordinates are $\frac{a \pi_{1}+c \pi_{1}^{\prime}}{n_{1}}, \frac{b \pi_{1}+a \pi_{1}^{\prime}}{n_{2}}$, respectively, with $a, b, c$, $d, n_{1}, n_{2} \in \boldsymbol{Z}$ and $n_{1} n_{2} \neq 0$. It follows that

$$
p\left(b^{* *}\right)=\pi_{i}^{\prime} b^{\prime}=\tau_{i}\left(\pi_{i} b^{\prime}\right)=\tau_{i} p\left(b^{*}\right)=p\left(\tau_{i} b^{*}\right) .
$$

Since $V \subseteq L$ and $p$ is injective on $L, b^{* *}=\tau_{i} b^{*}$. Comparing first coordinates tells us that

$$
\frac{b \pi_{1}+a \pi_{1}^{\prime}}{n_{2}}=\frac{\tau_{i}\left(d \pi_{1}+c \pi_{1}^{\prime}\right)}{n_{1}} .
$$

If $d \pi_{1}+c \pi_{1}^{\prime}=0$, then $b^{\prime}=b^{*} / \pi_{i}$ is of the form desired for basis elements of $L$.

If $d \pi_{1}+c \pi_{1}^{\prime} \neq 0$, then

$$
\tau_{i}=\frac{n_{1} a \tau_{1}+n_{1} b}{n_{2} e \tau_{1}+n_{2} d}
$$

Now $n_{1} n_{2}(a d-b c) \neq 0$. In fact,

$$
\begin{aligned}
\tau_{i}=\frac{n_{1}}{n_{2}} \frac{a \tau_{1}+b}{c \tau_{1}+d}= & \begin{cases}\frac{n_{1}}{n_{2}} \frac{a}{c}+\frac{b c-a d}{c} \frac{1}{c \tau_{1}+d} & \text { if } e \neq 0, \\
\frac{n_{1}}{n_{2}} \frac{a}{d} \tau_{1}+\frac{b}{d} & \text { if } c=0\end{cases} \\
& =\left\{\begin{array}{lll}
\frac{n_{1}}{n_{2}} \frac{a}{c} & \text { if } c \neq 0 & \text { and } a d-b c=0, \\
\frac{n_{1}}{n_{2}} \frac{b}{d} & \text { if } \quad c=0 \text { and } a d-b c=0,
\end{array}\right.
\end{aligned}
$$

which would contradict the assumption that $\tau_{i}$ be non-real. By assumption (b) above, we must have $i=1$. Clearing denominators in our ex pression for $\tau_{i}$ shows that either $\tau_{1}$ is quadratic or else $c=0$. In either ease, $b^{*} / \pi_{1}$ is a basis element of the desired form.

Summing up,

$$
B=\left\{b^{*} \pi_{i}^{-1} \mid b^{*} \in V, p\left(b^{*}\right)=\pi_{i} b^{*}, b^{\prime} \in B^{\prime} \cap W_{i}\right\}
$$

is a basis for $I$ of the form desired.

If $L$ is as in the proposition, then it is defined by equations of a special form. In fact, let $B$ be a basis as in the proposition. For each $i=1, \ldots, n$, the set $B \cap W_{i}$ generates a subspace of $K_{i}^{m_{i}}$. This subspace is defined by equations with coefficients from $K_{i}$ and involving the variables corresponding to the $m_{i}$ coordinates of $W_{i}$. The set of all these equations for all values of $i$ defines the subspace $L$. Thus $L$ is defined by equations each of which involves variables corresponding to only one set of $m_{i}$ coordinates and has coefficients from $\bar{K}_{i}$.

The following is crucial to all applications.

Theorear 2. For $i=1, \ldots, m$, let $\wp_{i}(z)$ and $\zeta_{i}(z)$ be the Weierstrass functions corresponding to the fundamental period pair $\pi_{i 1}, \pi_{i 2}$ with period ratio $\tau_{i}=\pi_{i 2} / \pi_{i 1}$. Suppose that

$$
\tau_{j} \neq \frac{a \tau_{i}+b}{b \tau_{i}+d}
$$

whenever $i \neq j$ and $a, b, c, d \in \mathbf{Z}$ satisfy $a d-b c \neq 0$. Let $K_{m+1}=Q$ and define for $i=1, \ldots$, in

$$
K_{i}= \begin{cases}Q\left(\tau_{i}\right) & \text { if } \tau_{i} \text { is quadratic } \\ Q & \text { otherwise }\end{cases}
$$

For $i=1, \ldots, m+1$, let $y_{i 1}, \ldots, y_{i n_{i}} \in C\left[\left[t_{1}, \ldots, t_{B}\right]\right]$ be $K_{i}$-linearly independent power series without constant terms. Define $r$ and $r$ to be the ranks of the Jacobian matrices

$$
\left(\frac{\partial y_{i j}}{\partial t_{k}}\right)_{\substack{i=1, \ldots, m+1 \\ j=1, \ldots, n_{i} \\ k=1, \ldots, s}} \text { and }\left(\frac{\partial y_{i j}}{\partial t_{k}}\right)_{\substack{i=1, \ldots, m \\ j=1, \ldots, n_{i} \\ k=1, \ldots, s}}
$$

respectively, and let $t$ be the transcendence degree over $\boldsymbol{C}$ of the field obtained. by adjoining to $\boldsymbol{C}$ all the $\wp_{i}\left(y_{i j}\right)$. Then

(i) $t \geqslant r^{\prime}$.

(ii) The transcendence degree over $C$ of the field generated by all of the "power series"

is at least $\sum_{i=1}^{m+1} n_{i}+r$.

$$
y_{k j}, \wp_{i}\left(y_{i j}\right), e^{y_{m+1, j}}
$$


(iii) The transcendence degree over $\boldsymbol{C}$ of the field generated by all the "power series"

is at least $\sum_{i=1}^{m+1} n_{i}+r+t$.

$$
y_{k j}, \wp_{i}\left(y_{i j}\right), \zeta_{i}\left(y_{i j}\right), e^{y_{m+1, j}}
$$

COROLLARY 10. With the notation of the theorem, the transcendence degree of the field in part (iii) is at least $2 \sum_{i=1}^{m} n_{i}+n_{m+1}+r$ provided that at least one of the following conditions holds true.

(i) The $\wp_{i}\left(y_{i j}\right)$ for $i=1, \ldots, m ; j=1, \ldots, n_{i}$ are algebraically independent.

(ii) The transcendence degree of the field generated by the $y_{i j}$ for $i=1, \ldots, m ; j=1, \ldots, n_{i}$ is at most (i.e. equal to) $r^{\prime}$.

(iii) The $y_{i j}$ for $i=1, \ldots, m ; j=1, \ldots, n_{i}$ are all polynomiats.

Proof (of corollary). Condition (i) implies $t=\sum_{i=1}^{m} n_{i}$ and so part (iii) of the theorem applies. Since condition (iii) clearly entails condition (ii), it suffices to show that the condition (ii) implies condition (i). But this follows from part (ii) of the theorem with $n_{m+1}=0$ since in this case $r=r^{\prime}$.

Proof (of theorem). (i) Using the proposition, one can apply Corollary 9 to the algebraic group $A=A_{1} \times \ldots \times A_{m}$ where $A_{i}$ is the product of $n_{i}$ copies of the elliptic curve parametrized by $\left(\wp_{i}(z), \wp_{i}^{\prime}(z)\right)$. One concludes that the transcendence degree of the field generated over $C$ by the $y_{i j}$ and the $\wp_{i}\left(y_{i j}\right)$ for $i \leqslant m$ is at least $\sum_{i=1}^{m} n_{i}+r^{\prime}$. Thus $t \geqslant r^{\prime}$.

(ii) The proof is the same except that one uses the algebraic group $G=L \times A$ where $A$ is as above and $L$ is a product of $n_{m+1}$ copies of the multiplicative group.

(iii) Let $T$ be a set of pairs $(i, j)$ such that the $\wp_{i}\left(y_{i j}\right),(i, j) \in T$ form a transcendence base for the field generated over $C$ by all the $\wp_{i}\left(y_{i j}\right)$. We wish to apply Corollary $I$ to the differentials $\omega_{i}-d \int_{d} \omega_{i}$ of the form

(6)

$$
\left\{\begin{array}{l}
\omega_{i j}-d y_{i j}=\frac{d \wp_{i}\left(y_{i j}\right)}{\wp_{i}^{\prime}\left(y_{i j}\right)}-d y_{i j} \quad \text { for } i=1, \ldots, m \text { and } j=1, \ldots, n_{i}, \\
\bar{\omega}_{i j}-d \zeta_{i}\left(y_{i j}\right)=\frac{\wp_{i}\left(y_{i j}\right) d \wp_{i}\left(y_{i j}\right)}{\wp_{i}^{\prime}\left(y_{i j}\right)}-d \zeta_{i}\left(y_{i j}\right) \quad \text { for }(i, j) \in T
\end{array}\right.
$$

and the logarithmic differentials

$$
e^{-y_{m+1, j}} d e^{y_{m+1, j}} \quad \text { for } \quad j=1, \ldots, n_{m+1} .
$$

By Corollary 1 , in order to prove assertion (iii), it suffices to show that the above differentials (6) are $C$-linearly independent. If not, then let

$$
\sum_{i, j} a_{i j}\left(\omega_{i j}-d y_{i j}\right)+\sum_{(i, j) \in T} \beta_{i j}\left(\bar{\omega}_{i j}-d \zeta_{i}\left(y_{i j}\right)\right)=0
$$

where not all of the coefficients $a_{i j}, \beta_{i j} \epsilon C$ are zero.

For each pair $(i, j) \in T$, we must have $\beta_{i j}=0$. In fact, for fixed $(i, j) \epsilon T$, let $K$ be the algebraic elosure in $C((t))$ of the field generated over $C$ by all of the $\wp_{k}\left(y_{k l}\right)$ where $(k, l) \in T \backslash\{(i, j)\}$. All of the $\wp_{u}\left(y_{u v}\right)$ are algebraic over $K\left(\wp_{i}\left(y_{i j}\right)\right)$. The natural map ${ }^{\wedge}: \Omega_{C(t) / \sigma} \rightarrow \Omega_{C(t(t)) / K}$ applied to the above linear relation shows that there is a differential of the first kind $\omega$ defined over an algebraic extension of $K\left(\wp_{i j}\left(y_{i j}\right)\right)$ such that $\beta_{i j}\left(\bar{\omega}_{i j}\right)^{\wedge}-\omega$ is exact where $\left(\bar{\omega}_{i j}\right)^{\wedge}$ is the image of $\bar{\omega}_{i j}$ in $\Omega_{C((t)) / K}$. By Lemma 3 , there is a function $f \in K\left(\wp_{i}\left(y_{i j}\right), \wp_{i}^{\prime}\left(y_{i j}\right)\right)$ such that $\beta_{i j}\left(\frac{\omega_{i j}}{\omega^{\wedge}}-d f\right.$ is a differential of the first kind. But $K\left(\wp_{i}\left(y_{i j}\right), \wp_{i}^{\prime}\left(y_{i j}\right)\right)$ is the function field over $K$ of the elliptic curve associated with the periods $\omega_{i 1}, \omega_{i 2}$. For this curve every differential of the second kind can be represented uniquely as the sum of an exact differential and a linear combination of $\left(\bar{\omega}_{i j}\right)^{\wedge}$ and $\left(\omega_{i j}\right)^{\wedge}\left([14]\right.$, p. 169). It follows that $\beta_{i j}=0$.

Since all the $\beta_{i j}$ for $(i, j) \in T$ are zero, the linear relation is reduced to

$$
\sum_{i, j} \alpha_{i j}\left(\omega_{i j}-d y_{i j}\right)=0 \text {. }
$$

But then $\sum_{i, j} \alpha_{i j} \omega_{i j}=d\left(\sum_{i, j} y_{i j}\right)$ is an exact differential of the first kind and so is zero. Hence the $\omega_{i j}$, which are just a set of generators for the left invariant differentials of $A$, are linearly dependent over $C$. By the last proposition and Lemma 4, we have a contradiction.

\section{APPLICATIONS}

A. Normalization. Necessary and sufficient conditions for the algebraic independence of two Weierstrass elliptic functions have long been known ([6], pp. 316-317). They were used by T. Schneider in his ground-breaking studies on the transcendence of these functions ([19]; [20], Ghapter II, $\S 4)$. The criterion for two functions $\wp(z)$ and $\wp^{*}(\beta z)$ with fundamental periods $\omega_{1}, \omega_{2}$ and $\omega_{1}^{*}, \omega_{2}^{*}$, respectively, to be algebraically dependent over $C$ is that there be rational numbers $r_{11}, r_{12}, r_{21}$, $r_{22}$ such that

$$
\begin{aligned}
& \beta^{-1} \omega_{1}^{*}=r_{11} \omega_{1}+r_{12} \omega_{2}, \\
& \beta^{-1} \omega_{2}^{*}=r_{21} \omega_{1}+r_{22} \omega_{2} .
\end{aligned}
$$


When we form the period ratios

$$
\tau=\frac{\omega_{2}}{\omega_{1}} \text { and } \quad \tau^{*}=\frac{\omega_{2}^{*}}{\omega_{1}^{*}}
$$

the preceding condition implies that

$$
\tau^{*}=\frac{a \tau+b}{c \tau+d}
$$

for some $a, b, c, d \in Z$. The conditions on the period ratios in the main theorem of the preceding section thus prevent $\wp_{1}(z)$ and $\wp_{2}(\beta z)$ from being algebraically dependent for $\wp_{1}(z) \neq \wp_{2}(z)$. This precaution is necessary for a sharp lower bound on the number of algebraically independent functions since the other hypotheses concern the arguments of only one elliptic function at a time.

Actually condition (5) does not restrict the generality of application of Theorem 2 and is thus only a normalization requirement. For if one has at hand two functions $\wp(z)$ and $\wp^{*}(z)$ with

$$
\tau^{*}=\frac{a \tau+b}{c \tau+d}
$$

for some $a, b, c, d \in \boldsymbol{Z}$, then set

$$
\beta=\frac{\omega_{I}^{*}}{c \omega_{2}+d \omega_{1}}
$$

It follows that

$$
\beta^{-1} \omega_{2 ?}^{*}=a \omega_{2}+b \omega_{1}
$$

Thus by $(7), \wp^{*}(\beta z)$ and $\wp(z)$ are algebraically dependent over $C$. Hence for purposes of algebraic independence of functions over $C$, one may replace $\wp(z)$ everywhere by $\wp^{*}(\beta z)$. Te shall see in Appendix A that the corresponding replacement of $\zeta(z)$ by $\zeta^{*}(\beta z)$ is also permissible as long as $z$ and $\wp^{*}(\beta z)$ lie among the functions whose independence is being considered.

B. Precise conditions for algebraic independence. In the case which is most important for number theoretic transcendence results, the corollary to Theorem 2 yields exact eonditions for the algebraic independence of the functions involved.

THEOREM 3. For $i=1, \ldots, m$, let $\wp_{i}(z)$ and $\zeta_{i}(z)$ be the Weierstrass functions corresponding to the pair of fundamental periods $\omega_{i 1}, \omega_{i 2}$ with period ratio $\tau_{i}=\omega_{i 2} / \omega_{i 1}$. Suppose that

$$
\tau_{j} \neq \frac{a \tau_{i}+b}{c \tau_{i}+d}
$$

whenever $i \neq j$ and $a, b, c, d \in Z$ satisfy $a d-b c \neq 0$. Set $K_{m+1}=Q$, and define for $i=1, \ldots, m$,

$$
K_{i}= \begin{cases}Q\left(\tau_{i}\right) & \text { if } \tau_{i} \text { is quadratic, } \\ Q & \text { otherwise. }\end{cases}
$$

For $i=1, \ldots, m+1$, let $\alpha_{i 1}, \ldots, \alpha_{i n_{i}} \in C^{*}$. Then the Laurent series for all the functions

$$
z, \wp_{i}\left(\alpha_{i j} z\right), \zeta_{i}\left(\alpha_{i j} z\right), \exp \left(\alpha_{m+1, k} z\right)
$$

$1 \leqslant i \leqslant m, 1 \leqslant j \leqslant n_{i}, 1 \leqslant k \leqslant n_{m+1}$, are algebraically independent over $\boldsymbol{C}$ if and only if, for each $1 \leqslant i \leqslant m+1$,

$$
\left\{a_{i 1}, \ldots, a_{i n_{i}}\right\} \text { is } K_{i}^{-} \text {-linearly independent. }
$$

We shal see in Appendix A that if any of the last conditions fails, then the functions are already algebraically dependent over $Q\left(g_{i 2}, g_{i 3}\right)$ for some $1 \leqslant i \leqslant m$. Moreover we shall determine the coefficients involved in achieving the normalization (9).

The result with no complex multiplication, i.e. each $K_{i}=Q$, and without the Weierstrass $\zeta$ functions follows from a theorem of Kolchin ([11]).

Now the sufficiency of condition (10) follows immediately from the corollary to Theorem 2. So we must check only the necessity of (10) which will follow from the criterion (7) for the dependence of two Weierstrass elliptic functions and the fundamental formulas ([24], p. 437),

$$
\left(\wp_{i}^{\prime}(z)\right)^{2}=4\left(\wp_{i}(z)\right)^{3}-g_{i 2} \wp_{i}(z)-g_{i 3},
$$

with $g_{i 2}, g_{i 3} \in C$, and ([24], pp. 440-441),

(12) $\wp_{i}(x+y)= \begin{cases}-\wp_{i}(x)-\wp_{i}(y)+\frac{1}{4}\left(\frac{\wp_{i}^{\prime}(x)-\wp_{i}^{\prime}(y)}{\wp_{i}(x)-\wp_{i}(y)}\right)^{2}, & x \neq \pm y \bmod \Lambda_{i}, \\ -2 \wp_{i}(x)+\frac{1}{4}\left(\frac{\wp_{i}^{\prime \prime}(x)}{\wp_{i}^{\prime}(x)}\right)^{2} & x \equiv-y \bmod \Lambda_{i}, \\ \infty & x \equiv y \bmod \Lambda_{i},\end{cases}$ where $\Lambda_{i}=\boldsymbol{Z} \omega_{i 1}+\boldsymbol{Z} \omega_{i 2}$.

For if $\alpha_{i 1}, \ldots, \alpha_{i m_{i}}$ are $K_{i}$-linearly dependent, then we can write one of the numbers, say $\alpha_{i 1}$, as a non-trivial $K_{i}$-linear combination of the others:

$$
\alpha_{i \mathrm{I}}=a_{2} a_{i 2}+\ldots+a_{n_{i}} \alpha_{i n_{i}} .
$$

The above formulas show that $\wp_{i}\left(\alpha_{i 1} z\right)$ is an algebraic function over $\boldsymbol{Q}\left(g_{i 2}, g_{i 3}\right)$ of the functions

$$
\wp_{i}\left(a_{2} \alpha_{i 2} z\right), \ldots, \wp_{i}\left(a_{n_{i}} a_{i n_{i}} z\right) .
$$


Now whenever $a \epsilon K_{i}^{*}, \wp_{i}(a z)$ is algebraic over $\boldsymbol{C}\left(\wp_{i}(z)\right)$. For when $\omega_{1}^{*}=\omega_{1}$ and $\omega_{1}^{*}=\omega_{2}$ in $(7)$, we are requiring there only that (upon dividing by $\omega_{1}$, respectively $\left.\omega_{2}\right)$

$$
a^{-1}=r_{11}+r_{12} \tau, \quad a^{-1}=r_{21} \tau^{-1}+r_{22},
$$

for some $r_{11}, r_{12}, r_{21}, r_{22} \in Q$. When $a_{\epsilon} Q^{*},(13)$ is satisfied with $r_{12}=r_{21}=0$. When $K_{i}=Q\left(\tau_{i}\right)$, any non-zero $a \in K_{1}=Q\left[\tau_{i}\right]=Q\left[\tau_{i}^{-1}\right]$ also satisfies (13). Thus if $a_{j} \in K_{i}^{*}, \wp_{i}\left(a_{j} a_{i j} z\right)$ is an algebraic function of $\wp_{i}\left(a_{i j} z\right), j=2, \ldots, n_{i}$. Consequently $\wp_{i}\left(\alpha_{i 1} z\right)$ is an algebraic function of $\wp_{i}\left(\alpha_{i 2} z\right), \ldots, \wp_{i}\left(\alpha_{i n_{i}} z\right)$ over $\boldsymbol{C}$, as claimed.

K. Ramachandra ([18], Lemma 7, p. 83), also gave a criterion guaranteeing the algebraic independence over $\boldsymbol{C}$ of more than two Weierstrass elliptic functions. Using Kronecker's theorem on simultaneous approximations, Ramachandra proved, among other things, the following result:

THEOREM (Ramachandra). Let $\omega_{1} ; \omega_{1}^{\prime} ; \ldots ; \omega_{n}, \omega_{n}^{\prime}$, respectively be fundamental periods for the Weierstrass elliptic functions $\wp_{1}(z), \ldots, \wp_{n}(z)$, respectively. If

(a) $\omega_{i} \omega_{j}^{-1}$ is real for every $1 \leqslant i, j \leqslant n$ and

(b) $\left\{\omega_{1}^{-1}, \ldots, \omega_{n}^{-1}\right\}$ is $Q$-linearly independent,

then the functions $\wp_{1}(z), \ldots, \wp_{n}(z)$ are algebraically independent over $\boldsymbol{C}$.

Since the form of Ramachandra's result appears so different from that of Theorem 3 (which was claimed to furnish necessary and sufficient conditions), perhaps it would be instructive to see how Ramachandra's criterion can be deduced.

Proof of Ramachandra's Criterion. For a given index $i$, let $j_{1}=i, j_{2}, \ldots, j_{\tau}$ be all the indices for which there are rational numbers $a_{k}, b_{k}, c_{k}, d_{k}$ with

$$
\tau_{j_{k}}=\frac{a_{k} \tau_{i}+b_{k}}{c_{k} \tau_{i}+d_{k}}
$$

where $\tau_{j}=\omega_{j}^{\prime} / \omega_{j}$ (for $j_{1}$, let $a_{1}=d_{1}=1, b_{1}=c_{1}=0$ ). Letting

$$
\beta_{k}=\frac{\omega_{j_{k}}}{c_{k} \omega_{i}+d_{k} \dot{\omega}_{i}}, \quad \text { i.e. } \quad \omega_{j_{k}} \beta_{k}^{-1}=c_{k} \omega_{i}^{\prime}+d_{k} \omega_{i} \text {, }
$$

one also has

$$
\omega_{j_{k}}^{\prime} \beta_{k}^{-1}=a_{k c} \omega_{i}^{\prime}+b_{k} \omega_{i} .
$$

Note that $\beta_{k}^{-1}=\alpha_{k} \omega_{i} \omega_{j_{k}}^{-1}$ where $\alpha_{k}=c_{k} \tau_{i}+d_{k}$. By the criterion (7), the functions $\wp_{j_{k}}\left(\beta_{k} z\right)$ and $\wp_{i}(z)$ are algebraically dependent, and hence so are $\wp_{j_{k}}(z)$ and $\wp_{i}\left(z \beta_{k}^{-1}\right)$. Thus the following conditions are equivalent:

(i) $\wp_{j_{1}}(z), \ldots, \wp_{j_{\tau}}(z)$ are algebraically independent. (ii) $\wp_{i}(z), \wp_{i}\left(z \beta_{z}^{-1}\right), \ldots, \wp_{i}\left(z \beta_{r}^{-1}\right)$ are algebraically independent.

Theorem 3 shows that condition (ii) is equivalent to

(iii) $1, \beta_{2}^{-1}, \ldots, \beta_{r}^{-1}$ are $K_{i}$-linearly independent.

In the case $K_{i}=Q\left(\tau_{i}\right)$, the fact that $\beta_{k}^{-1}=\alpha_{k} \omega_{i} \omega_{j_{k}}^{-1}$ with $\alpha_{k} \in K_{i}$ shows that condition (iii) is equivalent to

(iv) $\omega_{i} \omega_{j}^{-1}=1, \omega_{i} \omega_{j_{2}}^{-1}, \ldots, \omega_{i} \omega_{j_{r}}^{-1}$ are $K_{i}$-linearly independent.

But if

$$
\sum_{k}\left(e_{k}+\tau_{i} f_{k}\right) \omega_{i} \omega_{j_{k}}^{-1}=0
$$

with $e_{k}, f_{k} \epsilon \boldsymbol{Q}$, then since $\tau_{\boldsymbol{i}} \boldsymbol{R}$ whereas each $\omega_{\boldsymbol{i}} \omega_{j}^{-1} \epsilon \boldsymbol{R}$, one actually has two equations

$$
\begin{aligned}
& \sum_{k} e_{k} \omega_{i} \omega_{j_{k}}^{-1}=0 \\
& \sum_{k} f_{k} \omega_{i} \omega_{j_{k}}^{-1}=0 .
\end{aligned}
$$

Hypothesis (b) of Ramachandra's criterion implies now that each $e_{k}$ and $f_{k}$ is zero.

If $K_{i}=Q$, we consider condition (iii) directly. If

$$
\sum_{k} e_{k} \beta_{k}^{-1}=0
$$

with $e_{k} \epsilon Q$, then we have

$$
\sum_{k}\left(e_{k} c_{k} \tau_{i}+e_{k} d_{k}\right) \omega_{i} \omega_{j_{k}}^{-1}=0
$$

Since each $\omega_{i} \omega_{j_{k}}^{-1}$ is real, but $\tau_{i}$ is not real, we again get two equations

$$
\sum_{k} e_{k} c_{k} \omega_{i} \omega_{j_{k}}^{-1}=0, \quad \sum_{k} e_{k} d_{k} \omega_{i} \omega_{j_{k}}^{-1}=0
$$

Hypothesis (b) shows that for each $k, e_{k} c_{k}=e_{k} d_{k}=0$. But since not both $c_{k}$ and $d_{k}$ can be zero, each $e_{k}=0$.

Thus whether $K_{i}=Q$ or not, we see that the hypotheses of Ramachandra's criterion imply that the above three equivalent conditions hold.

C. Applications to transcendental numbers. An important component of modern transcendence proofs is the algebraic independence of the functions used to construct an auxiliary function. Theorem 3 fills a gap in our previous knowledge about such independence and allows the deduction of new results about the transcendence of certain numbers. The results fall into four general patterns. 
Common notation. For $i=1, \ldots, m$, let $\wp_{i}(z)$ be a Weierstrass elliptic function with period ratio $\tau_{i}$, invariants $g_{i 2}$ and $g_{i 3}$, and corresponding Weierstrass zeta function $\zeta_{i}(z)$ such that for $i \neq j$,

$$
\tau_{j} \neq \frac{a \tau_{i}+b}{c \tau_{i}+d}
$$

for any $a, b, c, d \in \boldsymbol{Z}$ with $a d-b c \neq 0$. Furthermore define, for $i=1, \ldots, m$,

$$
K_{i}= \begin{cases}Q\left(\tau_{i}\right) & \text { if } \tau_{i} \text { is imaginary quadratic, } \\ Q & \text { otherwise }\end{cases}
$$

Finally let $k_{z^{\prime}}$ denote any field of transcendence type $\leqslant \tau^{\prime}$ (see Appendix B for details).

Common hypotheses. For $i=1, \ldots, m$, let the set of complex numbers $\left\{\alpha_{i 1}, \ldots, \alpha_{i m_{i}}\right\}$ be $K_{i}$-linearly independent, and set $P=\sum_{i=1}^{m} m_{i}$. Let $\left\{\gamma_{i 1}, \ldots, \gamma_{i n_{i}}\right\}$ be arbitrary complex numbers, $0 \leqslant n_{i} \leqslant m_{i}$, and set $Z=\sum_{i=1}^{m} n_{i}$.

Let $\left\{\alpha_{1}, \ldots, a_{E}\right\}$ be a set of $Q$-linearly independent complex numbers.

Let $\left\{\beta_{1}, \ldots, \beta_{R}\right\}$ also be a set of $Q$-linearly independent numbers.

A Convention. If all the functions $\wp_{i}\left(\alpha_{i j} z\right), \gamma_{i j^{\prime}} z+\zeta_{i}\left(\alpha_{i j^{\prime}} z\right)$ and $\exp \left(a_{d} z\right)$ have a common non-zero period, then set $\delta=1$. Otherwise set $\delta=0$.

(The ranges of the indices in the theorems will be $1 \leqslant i \leqslant m, 1 \leqslant j \leqslant m_{i}$, $1 \leqslant j^{\prime} \leqslant n_{i}, 1 \leqslant d \leqslant E$, and $\left.1 \leqslant r \leqslant R.\right)$

THEOREM 4. If $\tau^{\prime}(2 P+2 Z+E+R-\delta) \leqslant R(P+Z+E)$, with strict inequality in case $\tau^{\prime}=1$, then not all the following numbers are algebraic over $k_{x^{\prime}}$ :

$g_{i 2}, g_{i 3}, \gamma_{i}\left(\alpha_{i j} \beta_{r}\right), \zeta_{i}\left(\alpha_{i j^{j}}, \beta_{r}\right)+\gamma_{i j^{\prime}} \beta_{r}, \exp \left(\alpha_{d} \beta_{r}\right)$.

THEOREM 5. If $\tau^{\prime}(2 P+2 Z+E+R) \leqslant R(1+P+Z+E)$, with strict inequality in case $\tau^{\prime}=1$, then not all the following numbers are algebraic over $k_{r^{\prime}}$ :

$g_{i 2}, g_{i 3}, \wp_{i}\left(\alpha_{i j} \beta_{r}\right), \zeta_{i}\left(\alpha_{i j} \beta_{r}\right)+\gamma_{i j^{\prime}} \beta_{r}, \exp \left(\alpha_{d} \beta_{r}\right), \beta_{r}$.

THEOREM 6. If $\left(\tau^{\prime}-1\right)(2 P+2 Z+E+R-2-\delta) \leqslant(R-2)(P+Z+E-1)$ and $P \geqslant 1$, then not all the following numbers are algebraic over $k_{t^{\prime}}$ :

$g_{i 2}, g_{i 3}, \wp_{i}\left(\alpha_{i j} \beta_{r}\right), \zeta_{i}\left(\alpha_{i j^{\prime}} \beta_{r}\right)+\gamma_{i j^{\prime}} \beta_{r}, \exp \left(\alpha_{d} \beta_{r}\right), \alpha_{i j}, \alpha_{d}, \gamma_{i j}$.

THEOREM 7 . If $\left(\tau^{\prime}-1\right)(2 P+2 Z+E+R-2) \leqslant(R-2)(P+Z+E)$ and $P \geqslant 1$, then not all the following numbers are algebraic over $k_{\tau^{*}}$ :

$g_{i 2}, g_{i 3}, \wp_{i}\left(\alpha_{i j} \beta_{r}\right), \zeta_{i}\left(\alpha_{i j}, \beta_{r}\right), \exp \left(\alpha_{d} \beta_{r}\right), \alpha_{i j}, \alpha_{i}, \beta_{r}$.
These theorems follow directly from Theorem 3 and Proposition 2, $\S 5$ of [23]. The techniques which Waldschmidt uses to establish that proposition come from the work of Schneider [20], Chapter II, Ramachandra [18] and Lang [12], Chapter $\nabla$. When $\tau^{\prime}=1$, Schneider proved a much stronger version of the last two theorems. Ramachandra proved Theorems 4 and 5 in the case $\tau^{\prime}=1$. Lang invented the notion of transcendence type (see Appendix $B$ ). The above theorems give new results when $P+Z>2$. For example, Theorem 4 allows us to complete Corollary 3 of $[23], \S 5$ :

Propostrxon 1. Let $\wp(z)$ and $\wp^{*}(z)$ be W.eierstrass elliptic functions with algebraic invariants $g_{2}, g_{3}$ and $g_{2}^{*}, g_{3}^{*}$ respectively, and fundamental pairs of periods $\omega_{1}, \omega_{2}$ and $\omega_{1}^{*}, \omega_{2}^{*}$, respectively. Assume

$$
\frac{\omega_{2}^{*}}{\omega_{1}^{*}} \neq \frac{a \omega_{2}+b \omega_{1}}{c \omega_{2}+d \omega_{1}}
$$

for every $a, b, c, d \in \mathbf{Z}$ with $a d-b c \neq 0$. Let $\zeta(z)$ be the Weierstrass zeta function associated with $\wp(z)$. Let $\omega^{*}$ and $\omega=a_{1} \omega_{1}+a_{2} \omega_{2}$ with $a_{1}, a_{2} \in \boldsymbol{Z}$ be non-zero periods of $\wp^{*}(z)$ and $\wp(z)$, respectively. Set

$$
\eta=2 a_{1} \zeta\left(\frac{\omega_{1}}{2}\right)+2 a_{2} \zeta\left(\frac{\omega_{2}}{2}\right)
$$

and let the complex numbers $1, v_{1}, v_{2}$ be $Q$-linearly independent. Then at least one of the six values

$$
\wp\left(\omega v_{i}\right), \zeta\left(\omega v_{i}\right)-\eta v_{i}, \wp^{*}\left(\omega^{*} v_{i}\right), \quad i=1,2,
$$

\section{is finite and transcendental.}

Since the four main theorems here involve so many parameters, let us try to get a feeling for the strength of these results. The following chart lists, for given $R$, the minimal choices for $P, Z, E$, when $P>0$. satisfying the hypotheses of 'Theorem 4 in the case $\tau^{\prime}=1$, i.e. $k_{z^{\prime}}$ is an algebraic number field. $N$ will denote the maximal number of quantities nvolved in the conclusion of the theorem:

\section{Table 1}

$\tau^{\prime}=1, P=m$

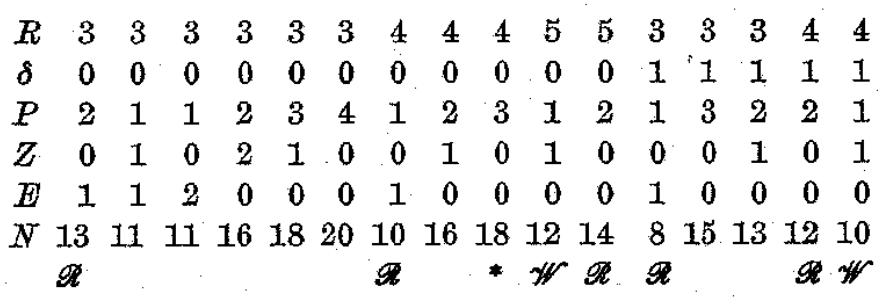


The letter $\mathscr{R}(\mathscr{W})$ in script under a column indicates that the case could be handled by Ramachandra's (Waldschmidt's) techniques alone.

Although $N$ is a general upper bound on the number of terms involved in the conclusion, in a particular application that bound may well be lowered. As an example, let us consider a corollary of the case (*) in Table 1 , where there are only 14 numbers involved instead of 18:

PRopostrion 2. Let $\wp(z)$ be a Weierstrass elliptic function with invariants $g_{2}, g_{3}$ and ratio of fundamental periods $\tau$, and let

$$
K= \begin{cases}Q(\tau) & \text { if } \tau \text { is quadratic } \\ Q & \text { otherwise. }\end{cases}
$$

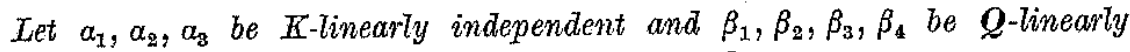
independent. Then at least one of the 14 numbers

$$
g_{2}, g_{3}, \wp\left(\alpha_{i} \beta_{j}\right), \quad i=1,2,3 ; j=1,2,3,4,
$$

is finite and transcendental.

If $\tau^{\prime} \neq 1$, then the Dirichlet box principle implies that $\tau^{\prime} \geqslant 2$. The following chart is the analogue of the above chart for $\tau^{\prime} \leqslant 2+\varepsilon$, when $\delta=0$, showing only the cases with $N \leqslant 41$.

\section{Table 2}

$\tau^{\prime} \leqslant 2+\varepsilon, \delta=0$

$\begin{array}{lllllllllllllllllll}R & 5 & 5 & 5 & 6 & 6 & 6 & 7 & 7 & 7 & 7 & 8 & 8 & 8 & 9 & 9 & 11 & 11\end{array}$

$\begin{array}{lllllllllllllllllll}P & 1 & 2 & 3 & 1 & 2 & 3 & 1 & 2 & 3 & 3 & 2 & 2 & 3 & 1 & 2 & 1 & 2\end{array}$

$\begin{array}{lllllllllllllllllll}Z & 1 & 0 & 2 & 0 & 1 & 0 & 1 & 2 & 1 & 2 & 0 & 1 & 0 & 0 & 2 & 1 & 0\end{array}$

$\begin{array}{llllllllllllllllll}E & 3 & 3 & 2 & 3 & 2 & 2 & 2 & 1 & 1 & 0 & 2 & 1 & 1 & 2 & 0 & 1 & 1\end{array}$

$\begin{array}{llllllllllllllllll}N & 27 & 29 & 41 & 26 & 34 & 36 & 30 & 39 & 41 & 41 & 36 & 36 & 38 & 29 & 40 & 35 & 37\end{array}$

Clearly the number of constants involved in the conclusion rises rather rapidly with $\tau^{\prime}$. However the less stringent inequalities of the other theorems allow us to deduce new results involving only a few numbers.

Proposition 3. Let $\wp(z)$ be a Weierstrass elliptic function having its period ratio $\tau=\omega_{2} / \omega_{1}$ imaginary quadratic and having its invariants al gebraic. Assume that the complex number $\alpha$ is a quartic with $[Q(\alpha, \tau): Q]=8$. Then for any complex number $\beta \neq 0$, at least one of the four numbers

$$
=\wp(\beta), \wp\langle\alpha \beta), \wp\left(\alpha^{2} \beta\right), \wp\left(\alpha^{3} \beta\right)
$$

is finite and transcendenta? over $Q(\pi)$.

Proof. Since $[Q(a, \tau): Q]=8,\left\{1, \alpha, a^{2}, a^{3}\right\}$ is linearly independent over $Q(v)$. We take $\delta=Z=E=0$ and $m=1$, but $P=3$ with $\left\{\alpha_{11}, \alpha_{12}\right.$, $\left.a_{13}, \alpha_{14}\right\}=\left\{\beta, \beta \alpha, \beta \alpha^{2}, \beta \alpha^{3}\right\}$ and the points $\beta_{r}$ given by the eight $Q$-linearly independent expressions $a^{i} \tau^{j}, i=0,1,2,3 ; j=0,1$. So $R=8$.
In Appendix A, we show that $\wp(z)$ and $\wp(\tau z)$ are algebraically dependent over $\boldsymbol{Q}\left(g_{2}, g_{3}\right)$. Since Feldman has shown ([5]) that $\boldsymbol{Q}(\pi)$ has transcendence type $\leqslant 2+\varepsilon$, for every $\varepsilon>0$, the result follows, by Theorem 5 .

Thus $\pi$ and at least one of the other three numbers mentioned in the conclusion of the proposition are algebraically independent. One can phrase the proposition in a more pointed form as follows:

PRopostrion 4. Let $\wp(z)$ and $\tau$ be as above. Let $\beta \in C$ be such that $\wp(\vec{\beta})$ is algebraic over $Q(x)$. Then at least one of the three values

$$
\wp(\alpha \beta), \wp\left(\alpha^{2} \beta\right), \wp\left(\alpha^{3} \beta\right)
$$

is finite and transcendental over $Q(\pi)$.

It follows from Feldman's main result in [32] that if $g_{2}$ and $g_{3}$ are algebraic and $\wp(z)$ has complex multiplications, then any non-zero period $\omega$ of $\wp(z)$ has transeendence type $\leqslant 2+\varepsilon$, for every $\varepsilon>0$. From this we can derive what seems to be the first known result about the algebraic independence of numbers related only to elliptic functions.

Propostrion 5 . Let $\wp(z)$ be any Weierstrass elliptic function with invariants $g_{2}, g_{3}$ algebraic and also having complex multiplications. If $\omega$ is any non-zero period of $\wp(z)$ and a any cubic irrational number, then at least one of the two numbers

$$
\wp(a \omega), \wp\left(a^{z} \omega\right)
$$

is transcendental over $\boldsymbol{Q}(\omega)$.

Feldman's transcendence measure ([31]) for $\omega$ when its elliptic function has no complex multiplications is $\leqslant 5+\varepsilon$ for every $\varepsilon>0$. The analogue of Proposition 5 would give only that one of the eight numbers $\wp\left(a^{i} \omega^{\prime}\right), 1 \leqslant i \leqslant 8$, will be transcendental over $\boldsymbol{Q}(\omega)$, when $[Q(\alpha, \tau): Q]$ $=18$ and $g_{2}^{\prime}, g_{3}^{\prime}$ are algebraic.

Many other such examples ean be constructed using the known transcendence types listed in Appendix D. For example, one can deduce the following result from Theorem 5 as above, using the fact that $\boldsymbol{Q}(e)$ has transcendence type $\leqslant 3([4])$.

PROPOSTTION 6. Let $\wp(z)$ and $\tau$ be as in Proposition 3 , but with $g_{2}$ and $g_{3}$ algebraic. Assume that $a \epsilon C$ is a quintic. Let $\beta$ be a non-zero compless number with $\wp(\beta)$ algebraic over $\boldsymbol{Q}(e)$. Then at least one of the four values

$$
\wp(\alpha \beta), \wp\left(\alpha^{2} \beta\right), \wp\left(\alpha^{3} \beta\right), \wp\left(\alpha^{4} \beta\right),
$$

is finite and transcendental over $Q(e)$. 


\section{APPENDIX A. THE CONSTANTS IN A DEPENDENCE RELATION}

The purpose of this section is to examine more closely the coefficients involved in normalizing the functions so that (9) holds, as indicated at the beginning of Section III, as well as the coefficients involved in an algebraic dependency arising from the failure to satisfy (10). The question is already interesting in itself. However the answer is crucial in applications to transcendental numbers. For example, the conclusions of Propositions 3 to 6 do not mention the constants involved in a dependence relation on $\wp(z)$ and $\wp(\tau z)$ since they are algebraic, as seen below. A corresponding result for $\zeta(z)$ is also proved.

In such applications, we shall always use the differential equations (11) directly or through the addition formala (12). Consequently we will always work over fields containing the constants $g_{2}$ and $g_{3}$ of the appropriate equations. If $\wp(z)$ is a Weierstrass elliptic function with lattice of periods $A$, in this section we shall write

$$
\wp(z)=\wp(z \mid \Lambda)
$$

To begin the section, we give a simple proof of a standard result.

Lemma. Let the coefficients of the differential equation for the Weierstrass function $\wp(z \mid \Lambda)$ be $g_{2}, g_{3}$. If $r$ is a non-zero rational number, then $\wp(z \mid \Lambda)$ and $\wp(r z \mid \Lambda)$ are algebraically dependent over $Q\left(g_{2}, g_{3}\right)$.

Proof. Say $r= \pm m / n, m, n \in \boldsymbol{N}$. Then repeated applications of the addition formula (12) and differential equation (11) together show that $\wp(m z \mid \Lambda)$ and $\wp(n z \mid \Lambda)$ are both algebraic over $Q\left(g_{2}, g_{3}, \wp(z \mid \Lambda)\right)$. Since neither is a constant, $\wp(m z \mid \Lambda)$ and $\wp(n z \mid \Lambda)$ are algebraically dependent over $Q\left(g_{2}, g_{3}\right)$. The change of variable $z \mapsto z / n$ shows that $\wp\left(\frac{m z}{n} \mid \Lambda\right)$ and $\wp(z \mid \Lambda)$ are algebraically dependent over $\boldsymbol{Q}\left(g_{2}, g_{3}\right)$. Since $\wp(z \mid \Lambda)$ is an even function, $\wp\left(\frac{m z}{n} \mid \Lambda\right)=\wp(r z \mid \Lambda)$.

As a consequence, one can, for example, derive the well-known result that $\wp(z \mid \Lambda)$ takes on values algebraic over $Q\left(g_{2}, g_{3}\right)$ at all division points of $A$, that is, points of $\frac{1}{n} A \backslash A$, for some $n \in \boldsymbol{N}$.

COROLLARY. Let $\alpha \in \frac{1}{n} \Lambda \backslash \Lambda$. Then $\wp(\alpha \mid \Lambda)$ is algebraic over $Q\left(g_{2}, g_{3}\right)$.

Proof. By the addition formula, we need only show this for $\omega_{1} / n$ and $\omega_{2} / n$ where $n>1$. But by the lemma, it is enough to show this when $n=2$, since $\wp(z \mid \Lambda)$ and $\wp\left(\frac{n}{2} z \mid \Lambda\right)$ are algebraically dependent over
$Q\left(g_{2}, g_{3}\right)$. It is clear from the definition ([24], p. 434),

$$
\wp(z \mid \mathcal{A})=z^{-2}+\sum_{\varpi \in \mathcal{A}}^{\prime}\left((z-\omega)^{-2}+\omega^{-2}\right)
$$

that $\wp^{\prime}(z) 1$ is an odd function of $z$ with period lattice 1 . Thus

$$
\wp^{\prime}\left(\frac{\omega_{i}}{2} \mid \Lambda\right)=-\wp^{\prime}\left(-\frac{\omega_{i}}{2} \mid \Lambda\right)=-\wp^{\prime}\left(\omega_{i}-\frac{\omega_{i}}{2} \mid \Lambda\right)=-\wp^{\prime}\left(\frac{\omega_{i}}{2} \mid \Lambda\right)
$$

and so

$$
\wp^{\prime}\left(\frac{\omega_{i}}{2} \mid \Lambda\right)=0 \quad \text { for } \quad i=1,2
$$

From the differential equation (11), we see that for $i=1,2$,

$$
0=\left(\wp^{\prime}\left(\frac{\omega_{i}}{2} \mid \Lambda\right)\right)^{2}=4\left(\wp\left(\frac{\omega_{i}}{2} \mid \Lambda\right)\right)^{3}-g_{2} \wp\left(\frac{\omega_{i}}{2} \mid \Lambda\right)-g_{3}
$$

PRoposimion. Let the Weierstrass elliptic functions $\wp(z \mid A)$ and $\wp\left(z \mid \Lambda^{*}\right)$ have lattices of periods $\Lambda=\boldsymbol{Z} \omega_{1}+\boldsymbol{Z} \omega_{2}$ and $\Lambda^{*}=\boldsymbol{Z} \omega_{1}^{*}+\boldsymbol{Z} \omega_{2}^{*}$, respectively; period ratios $\tau=\omega_{2} / \omega_{1}$ and $\tau^{*}=\omega_{2}^{*} / \omega_{1}^{*}$, respectively; and invariants $g_{2}, g_{\mathrm{s}}$ and $g_{2}^{*}, g_{3}^{*}$, respectively. Assume that

$$
\tau^{*}=\frac{a \tau+b}{c \tau+d}
$$

with $a, b, c, d \in \boldsymbol{Z}$ and $a d-b c \neq 0$. Then there is a number $\beta$, which is algebraic over $Q\left(g_{2}, g_{3}, g_{2}^{*}, g_{3}^{*}\right)$ such that the functions

$$
\wp(z \mid \Lambda), \wp\left(\beta z \mid \Lambda^{*}\right)
$$

are algebraically dependent over $Q\left(g_{2}, g_{8}, g_{2}^{*}, g_{3}^{*}\right)$.

Proof. Set $\beta=\omega_{1}^{*} /\left(e \omega_{2}+d \omega_{1}\right)$. Then it follows from (15) that $\beta$ is also $\omega_{2}^{*} /\left(a \omega_{2}+b \omega_{1}\right)$ and so $\Lambda^{*} \subseteq \beta \Lambda$. Select $0=\beta_{1}, \beta_{2}, \ldots, \beta_{m}$ to be a complete set of coset representatives for $\beta \Lambda$ modulo $A^{*}$. Then the function

$$
\wp(z \mid \beta \Lambda)-\sum_{i=1}^{m} \wp\left(z-\beta_{i} \mid \Lambda^{*}\right)
$$

at any point $\beta_{i}+\lambda, \lambda \in \Lambda^{*}$, has "infinite part"

$$
\left(z-\left(\beta_{i}+\lambda\right)\right)^{-2}-\left(\left(z-\beta_{i}\right)-\lambda\right)^{-2}=0
$$

Since these would be the only possible poles and since the function has periods $\Lambda^{*}$, it is bounded, and, by Liouville's theorem, it must be a constant $C$. Evaluation at $z=0$ gires

$$
C=\sum_{i=2}^{m} \wp\left(\beta_{i} \mid \Lambda^{*}\right) .
$$


Since $m \beta_{i} \in A^{*}$, for some positive integer $m$, the $\beta_{i}$ are division points of $A^{*}$. By the preceding corollary, each

$$
\wp\left(\beta_{i} \mid \Lambda^{*}\right), \quad i=2, \ldots, m,
$$

is algebraic over $\boldsymbol{Q}\left(g_{2}^{*}, g_{3}^{*}\right)$. Consequently upon making the change of variable $z \mapsto \beta z$ in (16) and applying the addition formula, we see that $\wp(\beta z \mid \beta A)$ is a rational function of $\wp\left(\beta z \mid \Lambda^{*}\right)$ with coefficients from a finite algebraic extension of $\boldsymbol{Q}\left(g_{2}^{*}, g_{3}^{*}\right)$. From the definition (14) of $\delta(z \mid \Lambda)$, it is obvious that

$$
\beta^{2} \wp(\beta \approx \mid \beta \Lambda)=\wp(\approx \mid \Lambda) .
$$

Thus we conclude that $\wp(z \mid \Lambda)$ and $\wp\left(\beta z \mid \Lambda^{*}\right)$ are algebraically dependent over $\boldsymbol{Q}\left(g_{2}^{*}, g_{3}^{*}, \beta\right)$.

To complete the proof, we recall $([24]$, p. 437$)$, that

$$
g_{2}=60 \sum_{\infty \in A}^{\prime} \omega^{-4}
$$

where the dash means that the term $\omega=0$ is omitted. Thus

$$
g_{2} \beta^{-4}=60 \sum_{\omega \in \mathcal{A}}^{\prime}(\beta \omega)^{-4}=60 \sum_{i=1}^{m} \sum_{\omega^{*} \in A^{*}}^{\prime}\left(\beta_{i}+\omega^{*}\right)^{-4}
$$

where the latter dash indicates that the term $\beta_{1}=0=\omega^{*}$ is omitted. When $i=1$, we have $\beta_{i}=0$ and

$$
60 \sum_{\infty^{*} \in \Lambda^{*}}^{\prime} \omega^{*-4}=g_{2}^{*} \text {. }
$$

When $i \neq 1, \beta_{i} \neq 0$, and

$$
60 \sum_{\omega^{*} \in \Lambda^{*}}^{\prime}\left(\beta_{i}+\omega^{*}\right)^{-4}=10 \beta^{\prime \prime}\left(\beta_{i} \mid \Lambda^{*}\right),
$$

as can be seen by differentiating (14) twice. But since, by (11),

$$
\wp^{\prime \prime}\left(z \mid A^{*}\right)=6\left(\wp\left(z \mid A^{*}\right)\right)^{2}-\frac{g_{2}^{*}}{2}
$$

and since $\wp\left(\beta_{i} \mid \Lambda^{*}\right), i \neq 1$, is algebraic over $Q\left(g_{2}^{*}, g_{3}^{*}\right)$, we can conclude that $g_{2} \beta^{-4}$ is algebraic over $Q\left(g_{2}^{*}, g_{3}^{*}\right)$. Consequently $\beta$ is algebraic over $Q\left(g_{2}^{*}, g_{3}^{*}, g_{2}\right)$, and the proposition is proven.

PRoposicion 7 . With the notation of Theorem 3 , suppose that $\left\{a_{i 1}, \ldots\right.$ $\left.\ldots, a_{i i_{i}}\right\}$ is $K_{i}$-linearly dependent. Then the functions $\wp\left(\alpha_{i 1} z \mid \Lambda_{i}\right), \ldots$ $\ldots, \wp\left(\alpha_{i n_{i}} z \mid \Lambda_{i}\right)$ are algebraically dependent over $\boldsymbol{Q}\left(g_{i 2}, g_{i 3}\right)$.
Proof. As the proof of Theorem 3 shows, the question hinges on whether $\wp\left(z \mid \Lambda_{i}\right)$ and $\wp\left(\alpha z \mid \Lambda_{i}\right)$ are algebraically dependent over $Q\left(g_{i q}, g_{i 3}\right)$ whenever $a \epsilon K_{i}$ is non-zero. The lemma above implies that dependence when $a \in Q$.

So we mas assume that $K_{i}=Q\left(\tau_{i}\right)$, with $\tau_{i}=(a+b \sqrt{-d}) / e$ where $a, b, c, d \epsilon \boldsymbol{Z}, d>0$. It is easy to check that with $\beta=2 c^{2} \tau_{i}$, we have $\beta \Lambda_{i} \subseteq \Lambda_{i}$. As in the preceding proof, let $0=\beta_{1}, \beta_{2}, \ldots, \beta_{m}$ be a complete set of coset representatives for $\Lambda_{i}$ modulo $\beta A_{i}$. Then

$$
\wp\left(z \mid \Lambda_{i}\right)=\sum_{i=1}^{m} \wp\left(z-\beta_{i} \mid \beta \Lambda_{i}\right)+C
$$

where each $\wp\left(\beta_{i} \mid \Lambda_{i}\right), i>1$, and hence $C$ are algebraic oxer $\boldsymbol{Q}\left(g_{i 2}, g_{i 3}\right)$. As before, the variable change $z \mapsto \beta z$ gives rise to

$$
\wp\left(\beta z \mid \Lambda_{i}\right)=\sum_{i=1}^{m} \wp\left(\beta z-\beta_{i} \mid \beta \Lambda_{i}\right)+C=\beta^{-2} \sum_{i=1}^{m} \wp\left(z-\frac{\beta_{i}}{\beta} \mid \Lambda_{i}\right)+C,
$$

where the last expression is a rational function in $\wp\left(z \mid \Lambda_{i}\right)$ with coefficients algebraic over $\boldsymbol{Q}\left(g_{i 2}, g_{i 3}\right)$.

Since every $a \in \boldsymbol{Q}\left(\tau_{i}\right)$ is of the form $\alpha=r+\delta \tau_{i}$, with $r, s \in \boldsymbol{Q}$, the general result follows from the special case $\beta=2 c^{2} \tau_{i}$ upon application of the addition formula (12) and the lemma of this section.

Propostryon 8. With the hypotheses of the preceding result, the functions

$$
\zeta\left(\alpha_{i 1} z \mid A_{i}\right), \ldots, \zeta\left(\alpha_{i n_{i}} z \mid A_{i}\right)
$$

are algebraically dependent over

$$
Q\left(g_{i 2}, g_{i 3}, \wp\left(\alpha_{i 1} z \mid \Lambda_{i}\right), \ldots, \wp\left(\alpha_{i i_{i}} z \mid \Lambda_{i}\right), z\right) .
$$

Proof. The proof when the coefficients of the linear dependency on $a_{i 1}, \ldots, \alpha_{i n_{i}}$ are from $\boldsymbol{Q}$ follows from the addition formula ([6], p. 297]):

$$
\zeta(x+y)= \begin{cases}\zeta(x)+\zeta(y)+\frac{1}{2}\left(\frac{\wp^{\prime}(x)-\wp^{\prime}(y)}{\wp(x)-\wp(y)}\right), & x \neq \pm y \bmod \Lambda_{i}, \\ 2 \zeta(x)+\frac{1}{2} \frac{\wp^{\prime \prime}(x)}{\wp^{\prime}(x)}, & x \equiv y \bmod \Lambda_{i}, \\ \infty, & x \equiv-y \bmod \Lambda_{i} .\end{cases}
$$

When $K_{i}=\boldsymbol{Q}\left(\tau_{i}\right)$, the question hinges on whether $\zeta\left(z \mid \Lambda_{i}\right)$ and $\zeta\left(\alpha z \mid \Lambda_{i}\right)$ are algebraically dependent over $\boldsymbol{Q}\left(g_{i 2}, g_{i 3}, \wp\left(z \mid \Lambda_{i}\right), z\right)$, for every non-zero $\alpha \in \mathbb{K}_{i}$. Again it suffices to check this for any particular ir- 
rational $\beta$ from $K_{i}$. Choose $\beta=20^{2} \tau_{i}$ as before, so that $\beta \Lambda_{i} \subseteq \Lambda_{i}$. Ohoose a complete set of coset representatives as follows:

$$
\begin{aligned}
& \lambda_{1}=0 \\
& \lambda_{2}=\frac{1}{2} \beta \omega_{1}, \\
& \lambda_{3}=\frac{1}{2} \beta \omega_{2}, \\
& \lambda_{4}=-\frac{1}{2}\left(\beta \omega_{1}+\beta \omega_{2}\right)
\end{aligned}
$$

and for $k$ odd, $4<k<m$,

$$
\lambda_{k}=-\lambda_{k+1}
$$

This is possible because $\beta \omega_{1} / 2, \beta \omega_{2} / 2, \beta\left(\omega_{1}+\omega_{2}\right) / 2$ represent the three cosets of order two. Then as above

$$
\wp\left(\beta z \mid \Lambda_{i}\right)=\beta^{-2} \sum_{k=1}^{m} \wp\left(z-\frac{\lambda_{k}}{\beta} \mid \Lambda_{i}\right)+C
$$

where $\beta^{-2} \wp\left(\frac{\lambda_{k}}{\beta} \mid \Lambda_{i}\right)=\wp\left(\lambda_{k} \mid \beta \Lambda_{i}\right), i>1$, and hence

$$
C=\sum_{k=2}^{m} \wp\left(\lambda_{k} \mid \beta \Lambda_{i}\right)
$$

are algebraic over $\boldsymbol{Q}\left(g_{i 2}, g_{i 3}\right)$. Since $([22]$, p. 445$)$,

$$
\zeta^{\prime}\left(z \mid \Lambda_{i}\right)=-\wp\left(z \mid \Lambda_{i}\right)
$$

integrating the expression in (17) gives us

$$
\beta^{-1} \zeta\left(\beta z \mid \Lambda_{i}\right)=\beta^{-2} \sum_{k=1}^{m} \zeta\left(z-\frac{\lambda_{k}}{\beta} \mid \Lambda_{i}\right)-C z+D
$$

We can evaluate $D$ by evaluating the functions at the origin:

$$
\left.D=-\beta^{-2} \sum_{k= \pm 2}^{m} \zeta\left(\frac{\lambda_{k}}{\beta}\right) \Lambda_{i}\right)
$$

Since $\zeta\left(z \mid \Lambda_{i}\right)$ is an odd function of $z$, we have

$$
\zeta\left(\frac{\lambda_{k}}{\beta} \mid \Lambda_{i}\right)=-\zeta\left(\frac{\lambda_{k+1}}{\beta} \mid \Lambda_{i}\right)
$$

when $k$ is odd and $4<k<m$. So in fact only the first three terms of (19) could contribute toward $D$ being non-zero. But by the definition of $\lambda_{4}$,

$$
\begin{aligned}
\zeta\left(\frac{\lambda_{i}}{\beta} \mid \Lambda_{i}\right) & =\zeta\left(-\frac{\omega_{1}+\omega_{2}}{2} \mid \Lambda_{i}\right)=-\zeta\left(\frac{\omega_{1}+\omega_{2}}{2} \mid \Lambda_{i}\right) \\
& =-\zeta\left(\frac{\omega_{1}}{2} \mid \Lambda_{i}\right)-\zeta\left(\frac{\omega_{2}}{2} \mid \Lambda_{i}\right)=-\zeta\left(\frac{\lambda_{2}}{\beta} \mid \Lambda_{i}\right)-\zeta\left(\frac{\lambda_{3}}{\beta} \mid \Lambda_{i}\right) .
\end{aligned}
$$

Thus $D=0$. Moreover, by the proof of the preceding result, both $\wp\left(\frac{\lambda_{k}}{\beta} \mid \Lambda_{i}\right)$ and $\wp^{\prime}\left(\frac{\lambda_{k}}{\beta} \mid \Lambda_{i}\right)$ are algebraic over $Q\left(g_{i 2}, g_{i 3}\right)$. The claimed result now follows upon applying the addition formula to equation (18).

\section{APPENDIX B. LTNEAR DEPENDENCE OF PERIODS AND QUASI-PERIODS}

Let $A=Z \omega_{1}+Z \omega_{2}, \tau=\omega_{2} / \omega_{1}, \zeta\left(z+\omega_{i} \mid A\right)=\zeta(z \mid \Lambda)+\eta_{i}, i=1,2$. THEOREM 8 . Let $\tau$ be imaginary quadratic. Then there is a complex number a algebraic over $Q\left(g_{2}, g_{3}\right)$ with

$$
\eta_{2}=\tilde{i} \eta_{1}+\alpha \omega_{1}
$$

where $\bar{\tau}$ is the complex conjugate of $\tau$.

Until recently such a result was known only when $g_{2}$ or $g_{3}$ was zero, in which cases $\alpha=0$. When $g_{2}$ and $g_{3}$ are algebraic, T. Schneider ([8], Satz 15, p. 60) has shown that $\omega_{1} / \eta_{1}$ is transcendental. Thus our theorem gives the only (up to multiplication by a constant) linear dependence relation on $\omega_{1}, \eta_{1}, \eta_{2}$ over the algebraie numbers when $g_{2}$ and $g_{3}$ are algebraic.

Building on the earlier work of A. Baker ([26], [27]) and J. Coates ([28], [29], [30]), D. W. Masser has very recently obtained many striking results on the linear independence over the algebraic numbers of algebraic points of $\wp(z \mid \Lambda)$. In particular, he obtained our theorem independently through a different but equally short proof. Moreover, using methods from the theory of elliptic modular functions, he is able to give a practical calculation method for $\alpha$ when $g_{2}$ and $g_{3}$ are algebraic.

Proof of theorem. From equation (17) and the addition formula for $\zeta(z \mid \Lambda)$, one has that the function

$$
f(z)=m \zeta(z \mid \Lambda)-\beta \zeta(\beta z \mid \Lambda)-\beta^{2} C z
$$

is an elliptic function. Replacing $z$ by $z+\omega_{1}$ shows that

$$
m \eta_{1}-2 C^{2} \beta \eta_{2}-\beta^{2} C \omega_{1}=0 \text {. }
$$


Now $m$ is the index of $\beta A$ in $A$, which is the determinant of multiplieation by $|\beta|$. Thas $m=|\beta|^{2}$. Substituting this and $\beta=2 O^{2} \tau$ into the above relation gives the result.

\section{APPENDIX O. WELERSTRASS STGMA FUNOTIONS}

Corollary 10 can be extended to include Weierstrass sigma functions and certain functions related to them. Recall ([24], p. 447) that the sigmar function is defined by

$$
\sigma(z)=z \prod_{\omega \in A}^{\prime}\left(1-\frac{z}{\omega}\right) e^{\left.\frac{z}{\omega}+\frac{1}{2} \frac{z}{2}\right)^{2}}
$$

where $A$ is a discrete two dimensional lattice in $C$, and that the logarithmic derivative of $\sigma(z)$ is just the Weierstrass zeta function $\zeta(z)$. We will be dealing with functions of the form

$$
g(z)=e^{q(z)} \sigma(z)
$$

where $q(z)$ is an algebraic function of $z$ analytic at the origin. This includes the sigma function as well as the fundamental theta function ([13], p. 259),

$$
f(z)=\frac{2 \pi i}{\omega_{2}} \eta^{2}\left(\frac{\omega_{2}}{\omega_{1}}\right) e^{-\eta_{1} 2^{2} / 2 \omega_{1}} \sigma(z)
$$

where $\omega_{1}, \omega_{2}$ is a pair of fundamental periods for $A$ and $\eta$ denotes the Dedekind eta function.

THEOREM 9. Let $\wp_{1}, \ldots, \wp_{m}, \zeta_{1}, \ldots, \zeta_{m}, \sigma_{1}, \ldots, \sigma_{m}$ be Weierstrass functions corresponding to the period pairs $\omega_{i 1}, \omega_{i 2}$ with period ratios $\tau_{i}=\omega_{i 2} / \omega_{i 1}$ where

$$
\tau_{i} \neq \frac{a \tau_{j}+b}{a \tau_{j}+d}
$$

for all $i \neq j$ and $a, b, c, d \in \mathbf{Z}$ with $a d-b c \neq 0$. For $1 \leqslant i \leqslant m$, let $g_{i}(z)$ $=e^{q_{i}(z)} \sigma_{i}(z)$ where $q_{i}(z)$ is an algebraic function of $z$ analytic at the origin. Let $K_{m+1}=Q$ and for $1 \leqslant i \leqslant m$, let

$$
K_{i}=\left\{\begin{array}{ll}
Q\left(\tau_{i}\right) & \text { if } \tau_{i} \text { is imaginary quadratic }, \\
Q & \text { otherwise. }
\end{array} .\right.
$$

Let $y_{i 1}, \ldots, y_{i n_{i}}, 1 \leqslant i \leqslant m+1$, be power series in $C\left[\left[t_{1}, \ldots, t_{s}\right]\right]$ without constant terms such that for each $i, y_{i 1}, \ldots, y_{i n_{i}}$ are $K_{i}$-tinearly independent.
Suppose that

$$
\begin{aligned}
& r=\operatorname{rank}\left(\frac{\partial y_{i j}}{\partial t_{k}}\right)_{\substack{i=1, \ldots, m \\
j=1, \ldots, m_{i} \\
k=1, \ldots, s^{s}}} \\
& =\text { tr. } \operatorname{deg} \cdot \boldsymbol{C} C\left(\left\{y_{i j} \mid i=1, \ldots, m ; j=1, \ldots, n_{i}\right\}\right) .
\end{aligned}
$$

Then the transcendence degree $T$ of the field generated over $C$ by all the functions

is precisely

$$
y_{i j}, \wp_{i}\left(y_{i j}\right), \zeta_{i}\left(y_{i j}\right), e^{y_{m+1, j}}, g_{i}\left(y_{i j}\right)
$$

$$
n_{m+1}+3 \sum_{i=1}^{m} n_{i}+r
$$

Proof. By Corollary 10, the transcendence degree of the field generated over $C$ by all of the functions

$$
y_{i j}, \wp_{i}\left(y_{i j}\right), \zeta_{i}\left(y_{i j}\right), e^{y_{m+1, j}}
$$

is precisely $n_{m+1}+2 \sum_{i=1}^{m} n_{i}+r$. Hence one need only show that $T$ is no smaller than the proposed value.

Let $J$ be a set of indices $(i, j)$ such that $\left\{y_{i j} \mid(i, j) \in J\right\}$ is a transcendence base over $C$ for the field generated by the $y_{i j}$ for $1 \leqslant i \leqslant m$ and $x \leqslant j \leqslant n_{i}$. Let $D_{1}, \ldots, D_{z}$ be linearly independent linear combinations of the partials $\partial / \partial t_{i}$ such that the matrix

$$
\left(D_{k} y_{i j}\right)_{k \leqslant(i, j) \in J}
$$

is the identity matrix, and $D_{k} y_{i j}=0$ for $(i, j) \in J$ and $k>\operatorname{Card} J$, the cardinality of the set $J$. For the construction of the $D_{k}$, see part (A) of the proof of Theorem 1 . For $1 \leqslant i \leqslant m, 1 \leqslant j \leqslant n_{i}, 1 \leqslant k_{i} \leqslant n_{m+1}$, and $(u, v) \in \mathcal{J}$, let

$$
\begin{aligned}
& \omega_{i j}=\frac{d \wp_{i}\left(y_{i j}\right)}{\wp_{i}^{\prime}\left(y_{i j}\right)}-d y_{i j} \\
& \omega_{i j}^{\prime}=\frac{\wp_{i}\left(y_{i j}\right) d \wp_{i}\left(y_{i j}\right)}{\wp_{i}^{\prime}\left(y_{i j}\right)}-d \zeta_{i}\left(y_{i j}\right), \\
& \omega_{i j}^{\prime \prime}=\frac{d g_{i}\left(y_{i j}\right)}{g_{i}\left(y_{i j}\right)}-\left(q_{i}^{\prime}\left(y_{i j}\right)+\zeta_{i}\left(y_{i j}\right)\right) \frac{d \wp_{i}\left(y_{i j}\right)}{\wp_{i}^{\prime}\left(y_{i j}\right)}, \\
& \omega_{k}^{(3)}=\frac{d e^{y_{j+1, k}}}{y_{m+1, k}}-d y_{m+1, k}, \\
& \omega_{u v}^{(4)}=d y_{u v} .
\end{aligned}
$$


If the conclusion of the theorem were false, then these $n_{m+1}+3 \sum_{i=1}^{m} n_{i}+r$ differentials would be linearly dependent. Choose a non-trivial equation of linear dependence involving the least possible number of $\omega_{i j}^{\prime \prime}$, say

$$
\sum_{i, j}\left(\alpha_{i j} \omega_{i j}+\beta_{i j} \omega_{i j}^{\prime}+\gamma_{i j} \omega_{i j}^{\prime \prime}\right)+\sum_{k} \delta_{k} \omega_{k}^{(3)}+\sum_{(u, v) \epsilon J} \varepsilon_{u v} \omega_{u v}^{(4)}
$$

By the first paragraph of the proof, at least one $\gamma_{i j}$ must be non-zero, and so by dividing by this coefficient, one can assume that at least one of the $\gamma_{i j}$ is one.

The differentials have been chosen so that

$$
D_{k}^{*} \omega_{i j}=D_{k}^{*} \omega_{i j}^{\prime}=D_{k}^{*} \omega_{i j}^{\prime \prime}=D_{k}^{*} \omega_{j}^{(3)}=0
$$

for all $i, j, k$. By the choice of the $D_{k}$ it follows by applying the $D_{k}^{*}$ to equation (20), that $\varepsilon_{u v}=0$ for all $(u, v) \in J$.

By the second lemma in the proof of Theorem 1 , one has for each $k$ with $1 \leqslant k \leqslant s$ that

$$
D_{k}^{1} \omega_{i j}=D_{k}^{\mathrm{I}} \omega_{i j}^{\prime}=D_{k}^{\mathrm{I}} \omega_{j}^{(3)}=0 .
$$

A straightforward computation shows that

$$
D_{k}^{1} \omega_{i j}^{\prime \prime}=-q_{i}^{\prime \prime}\left(y_{i j}\right) D y_{i j} \omega_{i j}+D y_{i j} \omega_{i j}^{\prime}
$$

where $D_{k}^{1}$ is as defined in part (C) of the proof of Theorem 1 .

Applying $D_{k}^{1}$ to equation (20) and simplifying yields

$$
\begin{aligned}
\sum_{i, j}\left\{\left(D_{k} \alpha_{i j}-q_{i}^{\prime \prime}\left(y_{i j}\right) \gamma_{i j} D_{k} y_{i j}\right) \omega_{i j}+\left(D_{k} \beta_{i j}+\gamma_{i j} D_{k} y_{i j}\right) \omega_{i j}^{\prime}+D_{k j} \gamma_{i j} \omega_{i j}^{\prime \prime}\right\}+ \\
+\sum_{j} D_{k} \delta_{j} \omega_{j}^{(3)}=0 .
\end{aligned}
$$

Since one of the $\gamma_{i f}$ was equal to 1 , this equation involves fewer of the $\omega_{i j}^{\prime \prime}$ and so by minimality is the trivial relation in light of Corollary 10. Thus

$$
\begin{aligned}
D_{k} \gamma_{i j}=D_{k} \delta_{j} & =0, \\
D_{k} \beta_{i j}+\gamma_{i j} D_{k} y_{i j} & =0, \\
D_{k} a_{i j}-\gamma_{i j} q_{i}^{\prime \prime}\left(y_{i j}\right) D_{k} y_{i j} & =0 .
\end{aligned}
$$

Since this holds for all $k$ with $1 \leqslant k \leqslant s$, it follows that $\gamma_{i j}$ and $\delta_{j}$ are constants and

$$
\begin{aligned}
& \beta_{i j}=-\gamma_{i j} y_{i j}+B_{i j}, \\
& \alpha_{i j}=\gamma_{i j} q_{i}^{\prime}\left(y_{i j}\right)+C_{i j},
\end{aligned}
$$
for some constants $B_{i j}$ and $C_{i j}$. Substituting back into equation (20)
gives

$$
\sum_{i, j}\left\{\left(\gamma_{i j} q_{i}^{\prime}\left(y_{i j}\right)+\sigma_{i j}\right) \omega_{i j}+\left(-\gamma_{i j} y_{i j}+B_{i j}\right) \omega_{i j}^{\prime}+\gamma_{i j} \omega_{i j}^{\prime i}\right\}+\sum_{j} \delta_{j} \omega_{j}^{(3)}=0 .
$$

Taking the exterior derivative of this last equation and using $d \omega_{i j}$ $=d \omega_{i j}^{\prime}=d \omega_{i j}^{(3)}=0$, one gets after simplifying that

$$
\begin{aligned}
\sum_{i, j} \frac{-\gamma_{i j} \wp_{i}\left(y_{i j}\right)}{\wp_{i}^{\prime}\left(y_{i j}\right)} d y_{i j} \wedge d \wp_{i}\left(y_{i j}\right)-\gamma_{i j} d y_{i j} \wedge d \zeta_{i}\left(y_{i j}\right)- \\
\quad-\frac{\gamma_{i j}}{\wp_{i}^{\prime}\left(y_{i j}\right)} d \zeta_{i}\left(y_{i j}\right) \wedge d \wp_{i}\left(y_{i j}\right)=0 .
\end{aligned}
$$

Now the $y_{i j}$ are algebraically dependent on the $y_{u v}$ with $(u, v) \in J$, and so the $d y_{i j} \wedge d \wp_{i}\left(y_{i j}\right)$ and $d y_{i j} \wedge d \zeta_{i}\left(y_{i j}\right)$ can be expressed as linear eombinations of the $d y_{u v} \wedge d \wp_{i}\left(y_{i j}\right)$ and $d y_{u v} \wedge d \zeta_{i}\left(y_{i j}\right)$ respectively. Substituting into the above equation gives an equation of linear dependence of the $d y_{u v} \wedge d \wp_{i}\left(y_{i j}\right), d y_{u v} \wedge d \zeta_{i}\left(y_{i j}\right)$, and $d \zeta_{i}\left(y_{i j}\right) \wedge d \wp_{i}\left(y_{i j}\right)$. But by the first paragraph of the proof, the $y_{u v}, \wp_{i}\left(y_{i j}\right), \zeta_{i}\left(y_{i j}\right)$ are algebraically independent, and so the relation must be trivial. Hence the $\gamma_{i j}$ are all zero, contrary to the choice of equation (20).

\section{APPENDIX D. TRANSCENDENCE TYPE}

The idea of the transcendence type of a field is due to Lang ([12] Chapter V). Although a general theory ([23], $\S 2$ ), has been developed when $k_{z^{\prime}}$ is a finitely generated extension of $Q$ lying in $C$ (see also [12], but beware of accepting the inequalities on pages $49,50,53,54$ at face value), in this appendix we will treat only the case that $k_{r^{\prime}}$ has transcendence degree one over $Q$, since so far the only fields with known transcendence types have transcendence degree one over $Q$.

For any non-zero polynomial $P(x) \in \mathbf{Z}[x]$, we define

$$
\text { size } P(x)=\max \{\operatorname{deg} P(x), \operatorname{loght} P(x)\},
$$

where ht $P(x)$ is the largest absolute value of any coefficient of $P(x)$.

If $k_{\tau^{\prime}}$ has transcendence degree one over $Q$, then we shall say that $k_{\tau^{\prime}}$ has transcendence type $\leqslant \tau^{\prime}$ if there is a transcendental number $\psi_{\epsilon} k_{\tau^{\prime}}$ and a constant $O>0$ such that for all non-zero $P(x) \in \mathbb{Z}[x]$,

$$
-O(\operatorname{size} P(x))^{x^{\prime}} \leqslant \log [P(x)] .
$$


It can be shown $([23], \S 2)$, that, in spite of appearances, this definition is independent of the transcendental number $x$ chosen. The following table lists the fields related to the exponential function known to us whose algebraic extensions have transcendence type $\leqslant \tau^{\prime}$, for some finite $\tau^{\prime}$.

\section{Table 3}

\begin{tabular}{|c|}
\hline $\begin{array}{c}k_{\tau^{\prime}} \\
Q(\pi)\end{array}$ \\
\hline$Q\left(e^{\pi}\right)$ \\
\hline $\boldsymbol{Q}\left(e^{\gamma}\right)$ \\
\hline $\begin{array}{c}Q(\log a) \\
Q\left(\alpha^{\beta}\right)\end{array}$ \\
\hline$Q\left(\frac{\log a}{\log s}\right.$ \\
\hline
\end{tabular}

$\begin{array}{cl}\tau^{\prime} & \text { Investigator } \\ 2+\varepsilon & \text { Feldman [5] } \\ 3+\varepsilon & \text { Cijsouw [4] } \\ 3 & \text { Cijsouw [4] } \\ 3+\varepsilon & \text { Cijsouw [4] } \\ 4+\varepsilon & \text { Gelfond [7] } \\ 4 & \text { Cijsouw [4] }\end{array}$

where $\gamma$ is any non-zero algebraie number, $\alpha$ is. algebraic with non-zero logarithm, $\beta$ is any irrational algebraic number, and $\delta$ is also algebraic with non-zero logarithm. Moreover Cijsouw has obtained transcendence types for fields of transcendence degree one generated by numbers which Baker's method shows to be transcendental ([4]). Cijsouw has also been able to remove the $\varepsilon$ from the type of $\alpha^{\beta}$ ([4]).

Added in proof: The authors are indebted to G. V. Choodnorsky for his helpful comments on the applications in III.C.

\section{References}

[1] J. Ax, On Schanuel's conjectures and Sholem's method, in Proceedings of Symposia in Pure Mathematice $X X$, Amer. Math. Soc., Providence, R. I., 1971.

[2] - On Schanuel's conjectures, Annals of Math. 93 (1971), pp. 252-268.

[3] - Some topios in differential algebraic geometry $I$ : Analytic subgroups of al. gebraic groups, Amer. J. Math. 94 (1972), pp. 1195-1204.

[4] P.L. Cijsouw, Transcendence Heasures, Thesis, University of Amsterdam, 1972.

[5] N.I. Feldman, On the measure of transcendence of $\pi$, Izv. Akad. Nauk SSSR, Ser. Mat. 24 (1960), pp. 357-368; Amer. Math. Soc. Transl. (2) 58 (1966), pp. 110-124.

[6] R. Fricke, Elliptische Funttionen, in Enoyklopädie der Mathematisehon Wissenschaften, II. B. 3, Teubner, Leipzig, 1901-1921.

[7] A. O. Gelfond, Transcendental and Algebraic Numbers, GITTL, Moscow 1952; Dover, New York 1960 .

[8] R. Gunning and H. Rossi, Analytic Functions of Several Complex Variables, Prentice-Hall, Englewood Cliffs, N. J., 1965.

[9] G. H. Hardy and E. M. Wright, An Introduction to the Theory of Numbers, 4th ed., Oxford University Press, Oxford 1960.

[10] $F$. Koizumi, On the differential forms of the first kind on algebraio varieties, J. Math. Soc. of Japan 2 (1949), pp. 273-280.
[11] E. Kolchin, Algebraic groups and algebraic dependence, Amer. J. Math. 90 (1968), pp. I15I-1164.

[12] S. Lang, Introduction to Transcendental Numbers, Addison-Wesley, Reading, Mass., 1965.

[13] - Elliptic Functions, Addison-Wesley, Reading, Mass., 1973.

[14] S. Lefschetz, Algebraic Geometry, Princeton University Press, Princeton, N. J., 1953.

[15] H. Matsumura, Oommutative Algebra, Benjamin, New Xork 1970.

[16] Y. Matsushima, Differential Manifolds, Marcel Dekker, New York 1972.

[17] D. Mumford, Introduction to Algebraic Geometry, Harvard Lecture Notes.

[18] K. Ramachandra, Contributions to the theory of transcendental numbers, $I, I I$, Acta Arith. 14 (1969), pp. 65-88.

[19] Th. Schneider, Arithmetische Untersuchungen elliptischer Integrale, Math. Ann. 113 (1937), pp. 1-13.

[20] - Einführung in die transzendenten Zahlen, Springer, Berlin 1957.

[21] Seminaire C. Chevalley, Classification des Groupes de Lie Algebriques, École Normale Supérieure, Paris 1958.

[22] G. Shimura and $Y$. Taniyama, Complex Multiplication of Abelian Varieties, Publ. Math. Soc. of Japan, Tokyo 1961.

[23] M. Waldschmidt, Propriétés arithmétiques des valeurs de fonctions méromorphes algébriquement indépendantes, Acta Arith. 23 (1973), pp. 19-88.

[24] E. T. Whittaker and G. N. Watson, A Course of Modern Analysis, 4th ed. Cambridge University Press, Cambridge 1927.

[25] O. Zariski, Local uniformization on algebraio varieties, Annals of Math. 41 (1940), pp. 852-896.

[26] A. B aker, On the periods of the Weierstrass fo-function, in Symposia Mathematica, Instituto Nationale Alta Matematica, Vol. IV, Rome 1970, pp. 155-174.

[27] - On the quasi-periods of the Weierstrass $\zeta$-function, Nachr. Akad. Wiss. Gottingen, Math. Phys. Klasse, Nr. 16 (1969), pp. 145-157.

[28] J. Coates, The transcendence of linear forms in $\omega_{1}, \omega_{2}, \eta_{1}, \eta_{2}, 2 \pi i$, Amer. J. Math. 113 (1971), pp. 385-397.

[29] - Linear forms in the periods of the exponential and elliptic functions, Inventiones Math. 12 (1971), pp. 290-299.

[30] - Linear relations betroeen $2 \pi i$ and the poriods of two elliptic ourves, in Diophantine Approximation and its Applications, Charles F. Osgood, ed., Acadeniio Press, New York 1973, pp. 77-99.

[31] N.I. Feldman, The approximation of certain transcendental numbers II, IzT. Akad. Nauk SSSR, Ser. Mat. 15 (1951), pp. 153-176; Amer. Math. Soc. Transi. (2) 59 (1966), pp. 246-270.

[32] - Simultaneous approximation of the periods of an elliptio function by algebraio numbers, Xzv. Akad. Nauk SSSR, Ser. Mat. 22 (1958), pp. 563-576; Amer. Math. Soc. Transl. (2) 59 (1966), pp. 271-284.

Received on 23. 4.19\%5

and in revised form on 30.12 .1975 